Article

\title{
Synthesis of a Novel Series of Amino Acid Prodrugs Based on Thienopyridine Scaffolds and Evaluation of Their Antiplatelet Activity
}

\author{
Nan Lu ${ }^{1,2}\left(\mathbb{D}\right.$, Lingjun $\mathrm{Li}^{2}$, Xuemin Zheng ${ }^{2}$, Shijun Zhang ${ }^{2}$, Yuquan $\mathrm{Li}^{2}$, Jing Yuan ${ }^{2}$, \\ Qunchao Wei ${ }^{2}$, Youjun $\mathrm{Xu}^{1, *}$ and Fancui Meng ${ }^{2, *(1)}$ \\ 1 School of Pharmaceutical Engineering, and Key Laboratory of Structure-Based Drug Design \& \\ Discovery (Ministry of Education), Shenyang Pharmaceutical University, Shenyang 110016, China; \\ lunantj@126.com \\ 2 Tianjin Key Laboratory of Molecular Design and Drug Discovery, Tianjin Institute of Pharmaceutical \\ Research, Tianjin 300193, China; lilj@tjipr.com (L.L.); zhengxm@tjipr.com (X.Z.); zhangsj@tjipr.com (S.Z.); \\ liyq@tjipr.com(Y.L.); yuanj@tjipr.com (J.Y.); weiqc@tjipr.com (Q.W.) \\ * Correspondence: xuyoujun@syphu.edu.cn (Y.X.); mengfc@tjipr.com (F.M.); Tel.: +86-24-4352-0223(Y.X.); \\ +86-22-2300-6833 (F.M.)
}

Received: 15 March 2018; Accepted: 26 April 2018; Published: 28 April 2018

\begin{abstract}
The thienopyridines class of drugs used as $\mathrm{P}_{2} \mathrm{Y}_{12}$ receptor antagonists plays a vital role in antiplatelet therapy. To further optimized this compound class, we designed and synthesized a series of amino acid prodrugs of 2-hydroxytetrahydrothienopyridine. All compounds were then evaluated for their inhibitory effect on ADP-induced platelet aggregation in rats and then $\mathrm{ED}_{50}$ and bleeding time of the most potent compounds were compared with commercial drugs. The results showed compound $\mathbf{5 c}$ could be a potent and safe candidate for further research.
\end{abstract}

Keywords: thienopyridines; $\mathrm{P}_{2} \mathrm{Y}_{12}$ receptor antagonist; antiplatelet; amino acid prodrugs; ADP-induced platelet aggregation

\section{Introduction}

Platelets play a critical role in the development of acute coronary syndromes (ACS) and contribute to cerebrovascular events through adhesion, aggregation and subsequent thrombus formation [1]. After platelet activation, ADP is released from intracellular storage granules and then further activates platelets, enlarging the activation and thus aggregation processes [2,3]. It was shown that the ADP response was due to activation of two receptors, the $\mathrm{G}_{\mathrm{q}}$-coupled $\mathrm{P} 2 \mathrm{Y}_{1}$ receptor, which induces a calcium response and shape change of the blood platelet and the $\mathrm{G}_{\mathrm{i}}$-coupled P2 $\mathrm{Y}_{12} \mathrm{R}$, which decreases the intracellular adenylyl cyclase activity and prolongs intracellular calcium signaling, thereby stabilizing the formed platelet aggregates [4-7]. Consequently, blocking the $\mathrm{P}_{2} \mathrm{Y}_{12} \mathrm{R}$ is a valid strategy to antiplatelet therapy, as demonstrated by the thienopyridine class of drugs, including clopidogrel and prasugrel (Figure 1). Thienopyridines are prodrugs those are converted into their respective active metabolites (AMs) through thiolactone intermediates. Clopidogrel is oxidized by cytochrome P450 (CYP) isoforms to its thiolactone, while prasugrel is rapidly hydrolysed by esterases to its thiolactone intermediate (Figure 2) [8]. Until now, dual antiplatelet therapy with aspirin and clopidogrel has remained the treatment of choice for patients with ACS and for those undergoing percutaneous coronary interventions [9-12]. However, up to $30 \%$ of Caucasian patients carry CYP2C19 loss-of-function alleles. They cannot complete the oxidative biotransformation after receiving clopidogrel and thus are prone to suffer a high rate of subsequent cardiovascular events [13,14]. Prasugrel achieved more pronounced inhibition of platelet aggregation 
and lower interindividual variability of pharmacological response, but the improved efficacy was associated with an increased bleeding risk [15]. Ticagrelor, unlike the thienopyridines, is an oral cyclopentyl-triazolopyrimidine (CPTP) that is a direct and reversible inhibitor of the $\mathrm{P}_{2} \mathrm{Y}_{12}$ receptor and does not require CYP450-mediated activation. Although it effectively reduces the ischaemic events and mortality rates of cardiovascular patients, high nonlethal bleeding rates are observed and obvious undesirable side-effect appear [16]. The TRITON and PLATO clinical trials have both accounted for the long-standing hypothesis of more potent platelet inhibition translating into reduced atherothrombotic events at the expense of increased bleeding [15-18]. All these considerations reinforce the strong need of novel and safe P2 $\mathrm{Y}_{12}$ antagonists.<smiles>COC(=O)[C@H](c1ccccc1Cl)N1CCc2sccc2C1</smiles>

clopidogrel

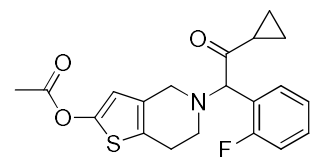

prasugrel

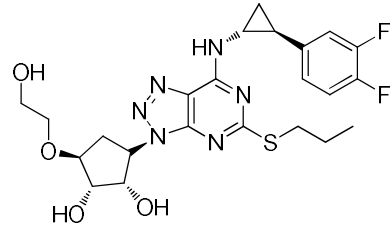

ticagrelor

Figure 1. The representative chemical structures of drugs of ADP receptor antagonist.

In this study, we mimicked the metabolic pattern of prasugrel and aimed to find a drug candidate overcoming the drawbacks of clopidogrel and prasugrel, assuming that ester prodrugs might be readily converted to thiolactones by esterase-mediated hydrolysis and subsequently to the active metabolite through only one CYP-dependent step. We chose the most potential intermediates 3a and $\mathbf{3 b}$ (Scheme 1) which are, respectively, the thiolactone of clopidogrel and prasugrel, as a parent part of the target compounds.

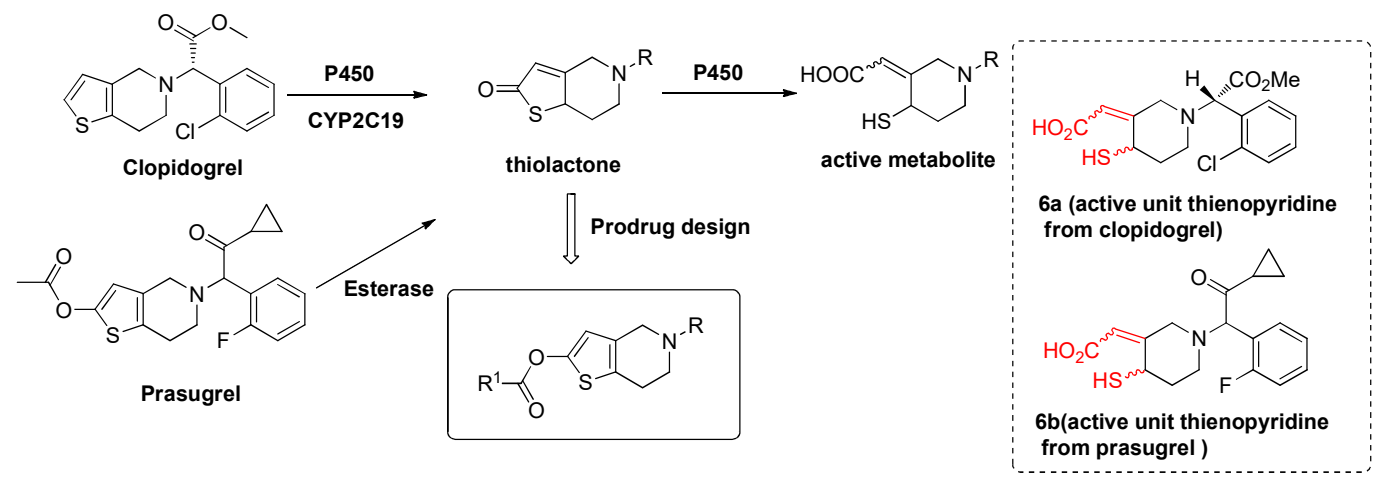

Figure 2. Simplified biosynthetic mechanism of the active units of thienopyridines and prodrug design.

Furthermore, amino acid prodrug design has been approached into our work as an attractive strategy. There are some good reasons for amino acid prodrugs: (1) they can be conveniently hydrolysed to their parent drug and amino acid part by enzymes in vivo. Amino acids are generally regarded as safe because they building blocks for proteins; (2) it is also proven that they can improve oral delivery and sustained release [19].

Taking the above information into account, we introduced several amino acids into the thiolactone moiety and synthesized a series of amino acid prodrugs of 2-hydroxytetra-hydrothienopyridine as novel antiplatelet agents. We also describe the inhibition of ADP-induced platelet aggregation in rats. Moreover, the potent compounds were tested for most $\mathrm{ED}_{50}$ and bleeding time. 


\section{Results}

\subsection{Chemistry}

The synthesis of the molecules was designed and carried out as shown in Scheme 1. The thienopyridine hydrochloride $\mathbf{1}$ was reacted with substituted intermediates $\mathbf{2} \mathbf{a}-\mathbf{b}$ in the absence of potassium bicarbonate to afford the $N$-alkylated thiolactones $3 \mathbf{a}-\mathbf{b}$, which were converted to $4 \mathbf{a}-4 \mathbf{p}$ with $N$-boc-L-amino acids via EDCI and DMAP $[20,21]$. We chose several aliphatic and aromatic amino acids, especially including the L-proline and L-2-pyrrolidone-5-carboxylic acid with an imine and amide structure, respectively. Finally, the target amino acid esters $5 a-5 p$ were obtained as hydrochloride salts after treating with hydrochloric acid in ethyl acetate for the removal of the Boc-group. All the structures are outlined in Table 1.

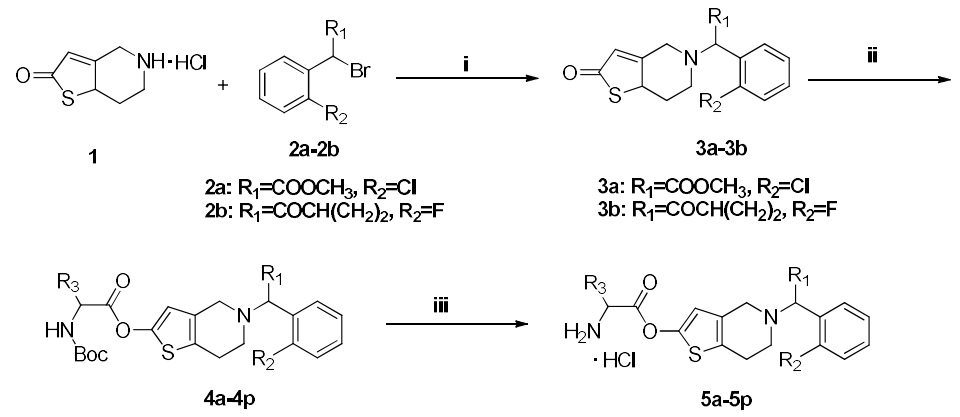

Scheme 1. Synthetic route of compounds 5a-5p. Reagents and conditions: (i) $\mathrm{K}_{2} \mathrm{CO}_{3}, \mathrm{CH}_{3} \mathrm{CN}$, r.t. overnight (60-65\% yield); (ii) N-Boc-L-amino acid, EDCI, DMAP, DCM, $0{ }^{\circ} \mathrm{C}$-r.t., 4 h $(71-86 \%$ yield); (iii) hydrochloric ethyl acetate, r.t., $5 \mathrm{~h}$ (84-93\% yield).

Table 1. Structures of target compounds.
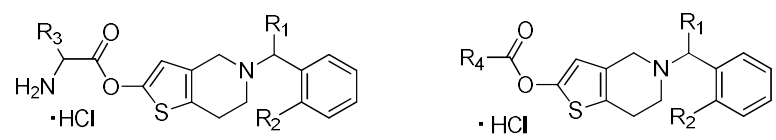

5a-5f, $5 i-5 n$

$5 g, 5 h, 50,5 p$

\begin{tabular}{|c|c|c|c|}
\hline & & & \\
\hline Compound & $\begin{array}{l}\mathbf{R}_{3} \text { or } \mathbf{R}_{4}{ }^{a} \\
\left(\mathbf{R}_{1}=\mathrm{COOCH}_{3}, \mathbf{R}_{2}=\mathrm{Cl}\right)\end{array}$ & Compound & $\begin{array}{l}\mathbf{R}_{3} \text { or } \mathrm{R}_{4}{ }^{\mathrm{a}} \\
\left(\mathrm{R}_{1}=\mathrm{COCH}\left(\mathrm{CH}_{2}\right)_{2}, \mathrm{R}_{2}=\mathrm{F}\right)\end{array}$ \\
\hline $5 a$ & $\mathrm{H}_{3} \mathrm{C} \cdot \xi_{-}$ & $5 \mathbf{i}$ & $\mathrm{H}_{3} \mathrm{C} \cdot \hat{-}-$ \\
\hline $5 b$ & $\underset{\text { ịn }}{\left(\mathrm{CH}_{3}\right)_{2} \mathrm{CH}}$ & $5 \mathbf{j}$ & 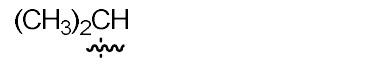 \\
\hline $5 c$ & $\left(\mathrm{CH}_{3}\right)_{2} \mathrm{CHCH}_{2}$ & $5 \mathrm{k}$ & $\left(\mathrm{CH}_{3}\right)_{2} \mathrm{CHCH}_{2}$ \\
\hline $5 d$ & $\mathrm{CH}_{3} \mathrm{CH}_{2} \mathrm{CH}\left(\mathrm{CH}_{3}\right)$ & 51 & $\mathrm{CH}_{3} \mathrm{CH}_{2} \mathrm{\sim}$ \\
\hline $5 e$ & & $5 \mathrm{~m}$ & \\
\hline $5 f$ & & $5 n$ & \\
\hline $5 g$ & & 50 & \\
\hline $5 \mathrm{~h}$ & & $5 p$ & \\
\hline
\end{tabular}

${ }^{a} R_{4}$ was only used for compounds $5 g-5 h$ and $5 o-5 p$. 


\subsection{Biological Activity Evaluation}

2.2.1. Inhibition of ADP-induced Platelet Aggregation in Rats at a Dose of $3 \mathrm{mg} / \mathrm{kg}$ and $1 \mathrm{mg} / \mathrm{kg}$

All the targeted analogues were evaluated for their inhibitory effect on ADP-induced platelet aggregation in rats, with clopidogrel and prasugrel as positive controls. The assay results are summarized in Table 2 and Figure 3. Considering that prasugrel exhibited strong potency at a dose of $3 \mathrm{mg} / \mathrm{kg}$, while clopidogrel was almost inactive, we initiated the screening at a dose of $3 \mathrm{mg} / \mathrm{kg}$ for all compounds. As we expected, almost all the first screening round compounds presented outstanding inhibitory effect on platelet aggregation that was more potent than clopidogrel. In particular, $\mathbf{5 c}$ and $5 \mathbf{i}-\mathbf{5 p}$ showed almost equal activity to that of prasugrel at $3 \mathrm{mg} / \mathrm{kg}$ dose. To make better option for further study, we attempted to increase the difference behavior among the targeted compounds on platelet aggregation via carrying out a secondary screening of all compounds at a reduced dose of $1 \mathrm{mg} / \mathrm{kg}$.

Table 2. Inhibitory effect of target compounds on ADP-induced platelet aggregation in rats at a dose of $3 \mathrm{mg} / \mathrm{kg}$ and $1 \mathrm{mg} / \mathrm{kg}$.

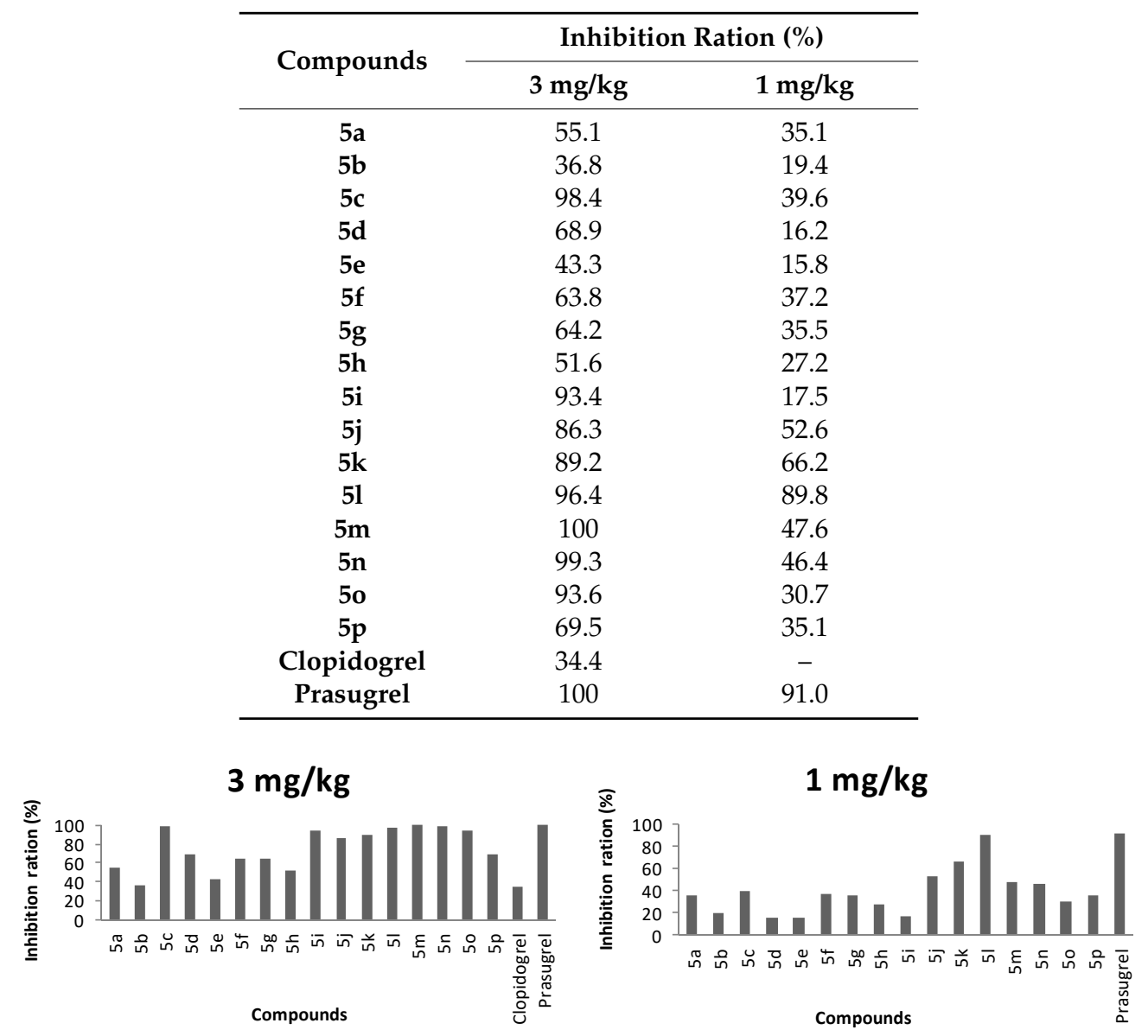

Figure 3. Inhibition ratios of all compounds at doses of $3 \mathrm{mg} / \mathrm{kg}$ and $1 \mathrm{mg} / \mathrm{kg}$.

As shown in the $1 \mathrm{mg} / \mathrm{kg}$ level results, $5 \mathbf{l}$ approached prasugrel in inhibition behavior and $\mathbf{5 c}, \mathbf{5 j}$, $5 \mathbf{k}, 5 \mathrm{~m}$ and $5 \mathrm{n}$ presented slightly less potency than prasugrel at the $1 \mathrm{mg} / \mathrm{kg}$ level. Although these compounds should covert to the same activate metabolites $\mathbf{6 a}$ or $\mathbf{6} \mathbf{b}$ to behave efficaciously [22,23], the hindrance of hydrolysis at the 2-ester moiety might have a significant impact on the potency. It was notable that 5c, based on the 2-oxoclopidogrel structure, was superior to clopidogrel and approach to prasugrel on inhibitory of platelet aggregation. 


\subsubsection{Determination of $\mathrm{ED}_{50}$ and $\mathrm{BT}_{2}$ of $5 \mathrm{c}$ and 51}

Aiming to find a candidate which has a balance between antiplatelet effects and bleeding complications, we selected $5 \mathrm{c}$ and 51 to further test for $\mathrm{ED}_{50}$ value and bleeding risk, because $5 \mathrm{c}$ and $\mathbf{5 l}$ performed the best in the aggregation assays among their own series of backbone structures which included 3a (2-oxoclopidogrel) and $\mathbf{3 b}$ (thiolactone of prasugrel), respectively. Firstly, we executed above bio-assay at doses of $0.5-4 \mathrm{mg} / \mathrm{kg}$ to determine the $\mathrm{ED}_{50}$ values of compounds $\mathbf{5 c}$ and 51 (Figure 4). The results showed that $5 \mathrm{c}\left(\mathrm{ED}_{50}=2.16 \mathrm{mg} / \mathrm{kg}\right)$ and $51\left(\mathrm{ED}_{50}=0.74 \mathrm{mg} / \mathrm{kg}\right)$ had a moderate value between clopidogrel $\left(\mathrm{ED}_{50}=3.96 \mathrm{mg} / \mathrm{kg}\right)$ and prasugrel $\left(\mathrm{ED}_{50}=0.50 \mathrm{mg} / \mathrm{kg}\right)$. Moreover, in the tail bleeding test (Figure 5), 5c induced much shorter bleeding time in rats, while $\mathbf{5 l}$ showed a slightly shorter bleeding time than prasugrel. Table 3 summarizes the $\mathrm{ED}_{50}$ and $\mathrm{BT}_{2}$ values for the tested compounds. The $\mathrm{BT}_{2}$ was defined as the dose that doubled the vehicle bleeding time. The $\mathrm{ED}_{50}$ and $\mathrm{BT}_{2}$ values of clopidogrel and prasugrel were in good agreement with those reported [24,25]. In addition, the ratios of $\mathrm{ED}_{50}$ to $\mathrm{BT}_{2}$ indicated the tested compounds have a similar benefit/bleeding ratio risk. It should be noted that the ratio of $\mathbf{5 c}$ was the lowest one, although its efficacy was lower than that of prasugrel. Taken together, these results suggest that $5 \mathrm{c}$ is a potent antiplatelet agent with relatively moderate antiheamostatic potency, but this remains to be proven in future clinical studies.

5c

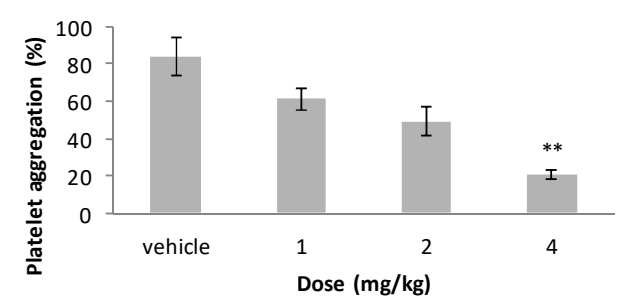

Clopidogrel

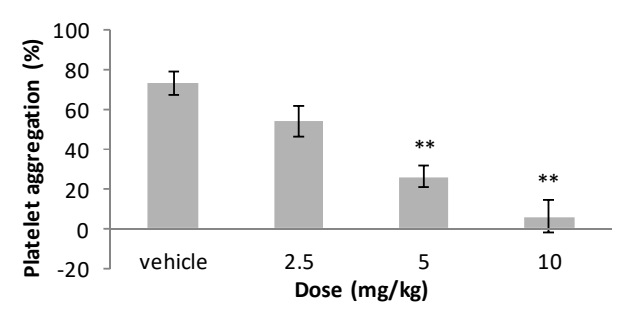

5

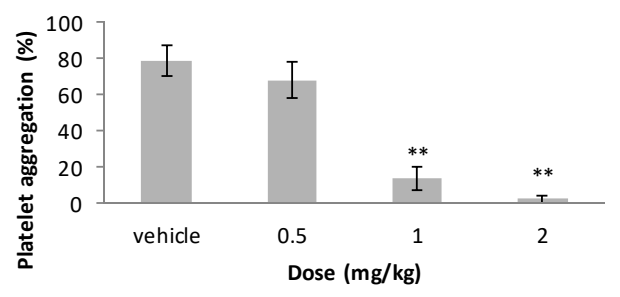

Prasugrel

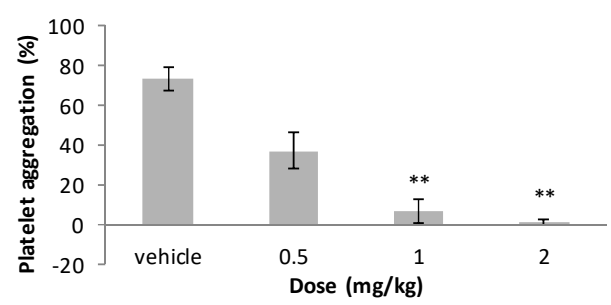

Figure 4. The platelet aggregation of $\mathbf{5 c}, 5 \mathbf{k}$, clopidogrel and prasugrel at different doses. ${ }^{* *} p<0.01$ vs. vehicle. Data are the mean $\pm \mathrm{SD}, n=5$ ). 
$5 c$

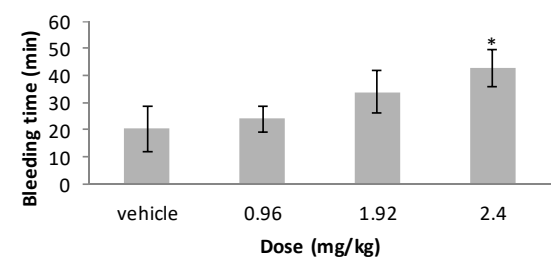

Clopidogrel

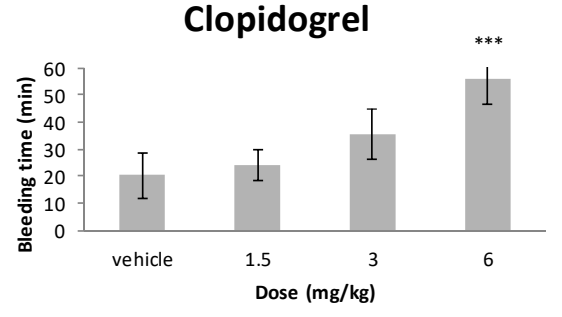

51

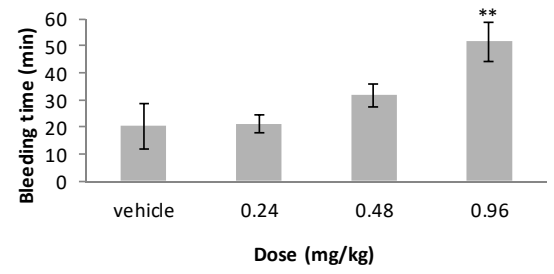

Prasugrel

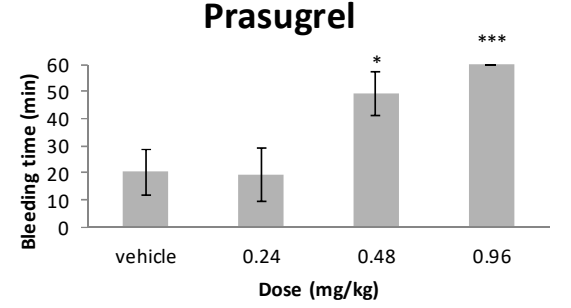

Figure 5. The bleeding time of $\mathbf{5 c}$ and $\mathbf{5 k}$ at different doses. ${ }^{*} p<0.05,{ }^{* *} p<0.01,{ }^{* * *} p<0.001$ vs. vehicle. Data are the mean $\pm \mathrm{SD}, n=5$ ).

Table 3. Comparison of antiplatelet antihaemostatic effects of potent compounds.

\begin{tabular}{cccc}
\hline Compounds & $\mathrm{ED}_{\mathbf{5 0}}(\mathbf{m g} / \mathbf{k g})$ & $\mathrm{BT}_{\mathbf{2}}(\mathbf{m g} / \mathbf{k g})$ & $\mathrm{ED}_{\mathbf{5 0}} / \mathbf{B T}_{\mathbf{2}}$ \\
\hline Clopidogrel & 3.96 & 3.82 & 1.04 \\
Prasugrel & 0.50 & 0.51 & 0.98 \\
$\mathbf{5 c}$ & 2.16 & 2.32 & 0.93 \\
$\mathbf{5 1}$ & 0.74 & 0.70 & 1.06 \\
\hline
\end{tabular}

\section{Discussion}

It has been reported that, at same dose, the activite metabolite of prasugrel (compound $\mathbf{6 b}$ ) is more potent at inhibiting platelet aggregation than that of clopidogrel (compound 6a) [26]. This very important point is exactly conformed by our study results wherebyt compounds $\mathbf{5 i - 5 p}$ generally were more effective than $\mathbf{5 a}-\mathbf{5 h}$, because of the generation of their respective AM after administration at the same dose. It is also indicated that $\mathbf{5 a}-\mathbf{5 h}$ can easier and more directly transform into $3 \mathbf{a}$ than clopidogrel via the evidence that $5 \mathbf{a}-\mathbf{5 h}$ are superior to clopidogrel while $5 \mathbf{i}-\mathbf{5 p}$ are not so to prasugrel. Taking all this into account, we believe that the compounds we designed and synthesized, especially $\mathbf{5 a}-\mathbf{5} \mathbf{h}$, are metabolized to $\mathbf{3 a}$ via a one-esterase hydrolysis-step and one-P450-step in vivo, which exactly overcomes the drawback of clopidogrel resistance. Further studies will focus on the pharmacokinetics of $\mathbf{5 c}$.

\section{Materials and Methods}

\subsection{General Information}

All reagents and solvents were purchased from commercial suppliers and used without further purification. Reactions were monitored by thin layer chromatography. The melting points (m.p.) of the compounds were determined on a YRT-3 Melting Point Tester (Precision Instrument of Tianjin University, Tianjin, China). ${ }^{1} \mathrm{H}-\mathrm{NMR}$ and ${ }^{13} \mathrm{C}-\mathrm{NMR}$ spectra were recorded for DMSO- $d_{6}$ solutions on a $400 \mathrm{MHz}$ Bruker spectrometer (Bruker, Billerica, MA, USA). MS were measured on a Finnigan LCQ Mass (Thermo Fisher Scientific, Waltham, MA, USA), HRMS were measured on a TOF LC/MS instrument (Agilent Technologies, Santa Clara, CA, USA). Blood sample were handled with a low speed table centrifuge (LD5-2A, Beijing, China) and an aggregometer (LBY-NJ4, Beijing, China). The ED 50 was calculated using the SPSS software (IBM, North Castle, NY, USA). 


\subsection{Chemistry}

Methyl 2-(2-chlorophenyl)-2-(2-oxo-7,7a-dihydrothieno[3,2-c]pyridin-5(2H,4H,6H)-yl)acetate (3a). To a stirred solution of methyl 2-bromo-2-(2-chlorophenyl)acetate $(2 \mathbf{a}, 26.3 \mathrm{~g}, 0.1 \mathrm{~mol})$ in $\mathrm{CH}_{3} \mathrm{CN}(500 \mathrm{~mL})$ were added 5,6,7,7a-tetrahydrothieno[3,2-c]pyridin-2(4H)-one hydrochloride (1, $20.9 \mathrm{~g}, 0.11 \mathrm{~mol})$ and potassium bicarbonate $(30.0 \mathrm{~g}, 0.3 \mathrm{~mol})$. The reaction was stirred at room temperature overnight. The reaction mixture was filtered and the liquid was concentrated under reduced pressure. The residue was purified by column chromatography to give a yellow oil, which was recrystallized from EtOH to afford a white solid (20.9 g, 62\% yield). m.p.: 118-119 ${ }^{\circ} \mathrm{C} .{ }^{1} \mathrm{H}-\mathrm{NMR}\left(400 \mathrm{MHz}, \mathrm{DMSO}-d_{6}\right): \delta 7.50-7.46$ $(\mathrm{m}, 2 \mathrm{H}), 7.41-7.35(\mathrm{~m}, 2 \mathrm{H}), 6.20(\mathrm{~s}, 1 \mathrm{H}), 4.85(\mathrm{~s}, 1 \mathrm{H}), 4.50(\mathrm{q}, 1 \mathrm{H}, J=6.0 \mathrm{~Hz}), 3.86(\mathrm{dd}, 1 \mathrm{H}, J=1.2 \mathrm{~Hz})$, $3.65(\mathrm{~s}, 3 \mathrm{H}), 3.22(\mathrm{~d}, 1 \mathrm{H}, J=12.0 \mathrm{~Hz}), 2.92(\mathrm{~d}, 1 \mathrm{H}, \mathrm{J}=12.0 \mathrm{~Hz}), 2.58(\mathrm{t}, 1 \mathrm{H}, J=12.0 \mathrm{~Hz}), 2.40-2.35(\mathrm{~m}$, $1 \mathrm{H}), 1.62-1.53(\mathrm{~m}, 1 \mathrm{H})$. ESI-MS $(\mathrm{m} / z)=338.16[\mathrm{M}+\mathrm{H}]^{+}$. The ${ }^{1} \mathrm{H}-\mathrm{NMR}$ and MS data were in good agreement with those reported [27].

5-(2-Cyclopropyl-1-(2-fluorophenyl)-2-oxoethyl)-5,6,7,7a-tetrahydrothieno[3,2-c]pyridin-2(4H)-one (3b). Compound $\mathbf{3 b}$ was synthesized from $\mathbf{2 b}$ and compound $\mathbf{1}$ according to the procedure described for the preparation of 3a. White solid product $\left(20.0 \mathrm{~g}, 65 \%\right.$ yield), m.p. $122-124{ }^{\circ} \mathrm{C} .{ }^{1} \mathrm{H}-\mathrm{NMR}(400 \mathrm{MHz}$, DMSO-d6): $\delta$ 7.37-7.31 (m, 2H), 7.24-7.10 (m, 2H), $6.03(\mathrm{~s}, 1 \mathrm{H}), 4.82(\mathrm{~s}, 1 \mathrm{H}), 4.07(\mathrm{q}, 1 \mathrm{H}, J=4.8 \mathrm{~Hz})$, $3.94(\mathrm{dd}, 1 \mathrm{H}, \mathrm{J}=12.0 \mathrm{~Hz}), 3.08-3.05(\mathrm{~m}, 2 \mathrm{H}), 2.35-2.30(\mathrm{~m}, 2 \mathrm{H}), 2.12-2.06(\mathrm{~m}, 1 \mathrm{H}), 1.93-1.83(\mathrm{~m}, 1 \mathrm{H})$, 1.04-1.01 (m, 2H), 0.90-0.78 (m, 2H). ESI-MS $(m / z)=332.19[\mathrm{M}+\mathrm{H}]^{+}$. The ${ }^{1} \mathrm{H}-\mathrm{NMR}$ and MS data were in good agreement with those reported [20].

5-(1-(2-chlorophenyl)-2-methoxy-2-oxoethyl)-4,5,6,7-tetrahydrothieno[3,2-c]pyridin-2-yl-2-((tert-butoxy-car bonyl)amino)propanoate (4a). EDCI $(1.2 \mathrm{~g}, 6 \mathrm{mmol})$ was slowly added to an ice-cooled mixture of 3a (1.0 g, $3 \mathrm{mmol})$, L-N-Boc-alanine (0.7 g, $3.6 \mathrm{mmol})$, DMAP (40 mg, $0.3 \mathrm{mmol})$ in DCM (15 mL). The mixture was gradually warmed to room temperature and stirred for additional $4.0 \mathrm{~h}$ until the completion of the reaction detected by TLC. Then it was poured into ice-water $(500 \mathrm{~mL})$. The organic was separated and the aqueous was extracted with DCM. The combined organic was successively washed with cold $1.0 \mathrm{M}$ aq $\mathrm{HCl}$, saturated aq $\mathrm{Na}_{2} \mathrm{CO}_{3}$, and brine. It was dried over $\mathrm{Na}_{2} \mathrm{SO}_{4}$ and vacuum evaporated to give $4 \mathrm{a}\left(1.2 \mathrm{~g}, 81.2 \%\right.$ yield) as a yellow oil. ${ }^{1} \mathrm{H}-\mathrm{NMR}\left(400 \mathrm{MHz}, \mathrm{DMSO}-d_{6}\right)$ : $7.55(\mathrm{~d}, 1 \mathrm{H}, J=7.8 \mathrm{~Hz}), 7.50-7.45(\mathrm{~m}, 2 \mathrm{H}), 7.38-7.32(\mathrm{~m}, 2 \mathrm{H}), 6.40(\mathrm{~s}, 1 \mathrm{H}), 4.82(\mathrm{~s}, 1 \mathrm{H}), 4.21-4.18(\mathrm{~m}, 1 \mathrm{H})$, $3.63(\mathrm{~s}, 3 \mathrm{H}), 3.52(\mathrm{~s}, 2 \mathrm{H}), 2.83-2.74(\mathrm{~m}, 2 \mathrm{H}), 2.66(\mathrm{~s}, 2 \mathrm{H}), 1.45-1.30(\mathrm{~m}, 12 \mathrm{H})$. ESI-MS $(\mathrm{m} / \mathrm{z})=509.08[\mathrm{M}+\mathrm{H}]^{+}$. Compounds $4 \mathrm{~b}-\mathbf{4} \mathrm{p}$ were synthesized according to the procedure described for the preparation of 4 a.

5-(1-(2-Chlorophenyl)-2-methoxy-2-oxoethyl)-4,5,6,7-tetrahydrothieno[3,2-c]pyridin-2-yl-2-((tert-butoxycarbonyl)-amino)-3-methylbutanoate (4b). Yellow oil. ${ }^{1} \mathrm{H}-\mathrm{NMR}\left(400 \mathrm{MHz}, \mathrm{DMSO}-d_{6}\right): \delta 7.55(\mathrm{~d}, 1 \mathrm{H}$, $J=7.2 \mathrm{~Hz}), 7.45(\mathrm{t}, 2 \mathrm{H}, J=8.0 \mathrm{~Hz}$ and $9.2 \mathrm{~Hz}), 7.38-7.31(\mathrm{~m}, 2 \mathrm{H}), 6.40(\mathrm{~s}, 1 \mathrm{H}), 4.81(\mathrm{~s}, 1 \mathrm{H}), 3.99(\mathrm{t}, 1 \mathrm{H}$, $J=6.8 \mathrm{~Hz}), 3.63(\mathrm{~s}, 3 \mathrm{H}), 3.52(\mathrm{~s}, 2 \mathrm{H}), 2.83-2.66(\mathrm{~m}, 4 \mathrm{H}), 2.10-2.05(\mathrm{~m}, 1 \mathrm{H}), 1.37(\mathrm{~s}, 9 \mathrm{H}), 0.92-0.89(\mathrm{~m}$, $6 \mathrm{H})$. ESI-MS $(m / z)=537.04[\mathrm{M}+\mathrm{H}]^{+}$.

5-(1-(2-Chlorophenyl)-2-methoxy-2-oxoethyl)-4,5,6,7-tetrahydrothieno[3,2-c]pyridin-2-yl-2-((tert-butoxy-car bonyl)amino)-4-methylpentanoate (4c). Yellow oil. ${ }^{1} \mathrm{H}-\mathrm{NMR}\left(400 \mathrm{MHz}, \mathrm{DMSO}-d_{6}\right): 7.55(\mathrm{~d}, 1 \mathrm{H}$, $J=6.8 \mathrm{~Hz}), 7.47(\mathrm{~d}, 2 \mathrm{H}, J=7.2 \mathrm{~Hz}), 7.39-7.32(\mathrm{~m}, 2 \mathrm{H}), 6.40(\mathrm{~s}, 1 \mathrm{H}), 4.83(\mathrm{~s}, 1 \mathrm{H}), 4.16-4.10(\mathrm{~m}, 1 \mathrm{H}), 3.63(\mathrm{~s}$, $3 \mathrm{H}), 3.52(\mathrm{~s}, 2 \mathrm{H}), 2.83-2.60(\mathrm{~m}, 4 \mathrm{H}), 1.65-1.40(\mathrm{~m}, 3 \mathrm{H}), 1.36(\mathrm{~s}, 9 \mathrm{H}), 0.88-0.84(\mathrm{~m}, 6 \mathrm{H})$. ESI-MS $(\mathrm{m} / z)=$ $551.05[\mathrm{M}+\mathrm{H}]^{+}, \mathrm{HRMS}(\mathrm{ESI})$ calcd. for $\mathrm{C}_{27} \mathrm{H}_{36} \mathrm{ClN}_{2} \mathrm{O}_{6} \mathrm{~S}^{+}:[\mathrm{M}+\mathrm{H}]^{+} m / z:$ 551.1977, found: 551.1968 .

5-(1-(2-Chlorophenyl)-2-methoxy-2-oxoethyl)-4,5,6,7-tetrahydrothieno[3,2-c]pyridin-2-yl-2-((tert-butoxy-car bonyl)amino)-3-methylpentanoate (4d). Yellow oil. ${ }^{1} \mathrm{H}-\mathrm{NMR}\left(400 \mathrm{MHz}, \mathrm{DMSO}-d_{6}\right): \delta 7.57(\mathrm{~d}, 1 \mathrm{H}$, $J=9.2 \mathrm{~Hz}), 7.48-7.34(\mathrm{~m}, 4 \mathrm{H}), 6.42(\mathrm{~s}, 1 \mathrm{H}), 4.83(\mathrm{~s}, 1 \mathrm{H}), 4.08-4.04(\mathrm{~m}, 1 \mathrm{H}), 3.65(\mathrm{~s}, 3 \mathrm{H}), 3.53(\mathrm{~s}, 2 \mathrm{H})$, 2.85-2.75 (m, 2H), 2.65-2.63 (m, 2H), 1.88-1.80 (m, 1H), 1.38-1.18 (m, 11H), 0.88-0.73 (m, 6H). ESI-MS $(\mathrm{m} / \mathrm{z})=551.10[\mathrm{M}+\mathrm{H}]^{+}$. 
5-(1-(2-Chlorophenyl)-2-methoxy-2-oxoethyl)-4,5,6,7-tetrahydrothieno[3,2-c]pyridin-2-yl-2-((tert-butoxy-car bonyl)amino)-3-phenylpropanoate (4e). Yellow oil. ${ }^{1} \mathrm{H}-\mathrm{NMR}\left(400 \mathrm{MHz}, \mathrm{DMSO}-d_{6}\right): \delta 7.38(\mathrm{t}, 2 \mathrm{H}$, $J=6.4 \mathrm{~Hz}), 7.31-7.29(\mathrm{~m}, 1 \mathrm{H}), 7.22-7.16(\mathrm{~m}, 2 \mathrm{H}), 7.12-7.03(\mathrm{~m}, 5 \mathrm{H}), 6.16(\mathrm{~s}, 1 \mathrm{H}), 4.66(\mathrm{~s}, 1 \mathrm{H}), 4.23-4.14$ $(\mathrm{m}, 1 \mathrm{H}), 3.47(\mathrm{~s}, 3 \mathrm{H}), 3.35(\mathrm{~s}, 2 \mathrm{H}), 3.26-3.10(\mathrm{~m}, 2 \mathrm{H}), 2.92-2.80(\mathrm{~m}, 2 \mathrm{H}), 2.67-2.57(\mathrm{~m}, 2 \mathrm{H}), 1.16(\mathrm{~s}, 9 \mathrm{H})$. $\operatorname{ESI-MS}(m / z)=585.11[\mathrm{M}+\mathrm{H}]^{+}$.

5-(1-(2-Chlorophenyl)-2-methoxy-2-oxoethyl)-4,5,6,7-tetrahydrothieno[3,2-c]pyridin-2-yl-2-((tert-butoxy-car bonyl)amino)-3-(1H-indol-2-yl)propanoate (4f). Yellow oil. ${ }^{1} \mathrm{H}-\mathrm{NMR}\left(400 \mathrm{MHz}, \mathrm{DMSO}-\mathrm{d}_{6}\right): \delta 10.86(\mathrm{~s}$, $1 \mathrm{H}), 7.59(\mathrm{~d}, 1 \mathrm{H}, J=8.0 \mathrm{~Hz}), 7.53-7.48(\mathrm{~m}, 3 \mathrm{H}), 7.39-7.33(\mathrm{~m}, 2 \mathrm{H}), 7.18(\mathrm{~s}, 1 \mathrm{H}), 7.06(\mathrm{t}, 1 \mathrm{H}, J=8.0 \mathrm{~Hz})$, $6.98(\mathrm{t}, 1 \mathrm{H}, J=8.0 \mathrm{~Hz}), 6.26(\mathrm{~s}, 1 \mathrm{H}), 4.83(\mathrm{~s}, 1 \mathrm{H}), 4.42-4.37(\mathrm{~m}, 1 \mathrm{H}), 3.65(\mathrm{~s}, 3 \mathrm{H}), 3.52(\mathrm{~s}, 2 \mathrm{H}), 3.19-3.12$ $(\mathrm{m}, 2 \mathrm{H}), 2.86-2.75(\mathrm{~m}, 2 \mathrm{H}), 2.66-2.58(\mathrm{~m}, 2 \mathrm{H}), 1.35(\mathrm{~s}, 9 \mathrm{H})$. ESI-MS $(\mathrm{m} / \mathrm{z})=624.17[\mathrm{M}+\mathrm{H}]^{+}$.

1-tert-Butyl 2-(5-(1-(2-chlorophenyl)-2-methoxy-2-oxoethyl)-4,5,6,7-tetrahydrothieno[3,2-c]pyridin-2-yl) pyrrolidine-1,2-dicarboxylate (4g). Yellow oil. ${ }^{1} \mathrm{H}-\mathrm{NMR}\left(400 \mathrm{MHz}, \mathrm{DMSO}-d_{6}\right): \delta 7.56(\mathrm{t}, 1 \mathrm{H}, \mathrm{J}=2.0 \mathrm{~Hz}$ and $6.8 \mathrm{~Hz}), 7.46(\mathrm{t}, 1 \mathrm{H}, J=13.6 \mathrm{~Hz}), 7.38-7.32(\mathrm{~m}, 2 \mathrm{H}), 6.44(\mathrm{~s}, 1 \mathrm{H}), 4.82(\mathrm{~s}, 1 \mathrm{H}), 4.37-4.34(\mathrm{~m}, 1 \mathrm{H})$, $3.63(\mathrm{~s}, 3 \mathrm{H}), 3.52(\mathrm{~s}, 2 \mathrm{H}), 3.41-3.27(\mathrm{~m}, 2 \mathrm{H}), 2.80-2.74(\mathrm{~m}, 2 \mathrm{H}), 2.66(\mathrm{~s}, 2 \mathrm{H}), 2.33-2.26(\mathrm{~m}, 1 \mathrm{H}), 1.98-1.94$ $(\mathrm{m}, 1 \mathrm{H}), 1.86-1.80(\mathrm{~m}, 2 \mathrm{H}), 1.35(\mathrm{~s}, 9 \mathrm{H})$. ESI-MS $(\mathrm{m} / \mathrm{z})=535.05[\mathrm{M}+\mathrm{H}]^{+}$.

1-tert-Butyl 2-(5-(1-(2-chlorophenyl)-2-methoxy-2-oxoethyl)-4,5,6,7-tetrahydrothieno[3,2-c]pyridin-2-yl) 5-oxopyrrolidine-1,2-dicarboxylate (4h). Yellow oil. ${ }^{1} \mathrm{H}-\mathrm{NMR}\left(400 \mathrm{MHz}, \mathrm{DMSO}-d_{6}\right): \delta 7.35(\mathrm{dd}, 1 \mathrm{H}$, $J=7.2 \mathrm{~Hz}), 7.28-7.25(\mathrm{~m}, 1 \mathrm{H}), 7.17-7.14(\mathrm{~m}, 2 \mathrm{H}), 6.30(\mathrm{~s}, 1 \mathrm{H}), 4.66-4.62(\mathrm{~m}, 2 \mathrm{H}), 3.43(\mathrm{~s}, 3 \mathrm{H}), 3.22(\mathrm{~s}, 2 \mathrm{H})$, 2.60-2.47 (m, 4H), 2.30-2.18 (m, 3H), 1.85-1.76 (m, 1H), $1.17(\mathrm{~s}, 9 \mathrm{H})$. ESI-MS $(\mathrm{m} / z)=549.05[\mathrm{M}+\mathrm{H}]^{+}$.

5-(2-Cyclopropyl-1-(2-fluorophenyl)-2-oxoethyl)-4,5,6,7-tetrahydrothieno[3,2-c]pyridin-2-yl-2-((tert-butoxycarbonyl)amino)propanoate (4i). Yellow oil. ${ }^{1} \mathrm{H}-\mathrm{NMR}\left(400 \mathrm{MHz}, \mathrm{DMSO}-d_{6}\right)$ : 7.52-7.49 (m, 1H), 7.40-7.38 $(\mathrm{m}, 2 \mathrm{H}), 7.27-7.22(\mathrm{~m}, 2 \mathrm{H}), 6.43(\mathrm{~s}, 1 \mathrm{H}), 4.78(\mathrm{~s}, 1 \mathrm{H}), 4.08-4.06(\mathrm{~m}, 1 \mathrm{H}), 3.48(\mathrm{~s}, 2 \mathrm{H}), 2.89-2.72(\mathrm{~m}, 4 \mathrm{H})$, 2.27-2.25 (m, 1H), 1.47-1.15 (m, 12H), 0.88-0.80 (m, 4H). ESI-MS $(m / z)=503.11[\mathrm{M}+\mathrm{H}]^{+}$.

5-(2-Cyclopropyl-1-(2-fluorophenyl)-2-oxoethyl)-4,5,6,7-tetrahydrothieno[3,2-c]pyridin-2-yl-2-((tert-butoxy-car bonyl)amino)-3-methylbutanoate (4j). White solid. m.p. 106-108 ${ }^{\circ} \mathrm{C} .{ }^{1} \mathrm{H}-\mathrm{NMR}\left(400 \mathrm{MHz}, \mathrm{DMSO}-d_{6}\right)$ : $\delta 7.52-7.45(\mathrm{~m}, 2 \mathrm{H}), 7.42-7.37(\mathrm{~m}, 1 \mathrm{H}), 7.26-7.22(\mathrm{~m}, 2 \mathrm{H}), 6.44(\mathrm{~s}, 1 \mathrm{H}), 4.78(\mathrm{~s}, 1 \mathrm{H}), 4.00(\mathrm{t}, 1 \mathrm{H}$, $J=6.4 \mathrm{~Hz}), 3.44(\mathrm{t}, 2 \mathrm{H}, J=16.0 \mathrm{~Hz}), 2.80-2.69(\mathrm{~m}, 4 \mathrm{H}), 2.38-2.36(\mathrm{~m}, 1 \mathrm{H}), 2.11-2.06(\mathrm{~m}, 1 \mathrm{H}), 1.38(\mathrm{~s}, 9 \mathrm{H})$, 0.94-0.78 (m, 10H). ESI-MS $(m / z)=531.22[\mathrm{M}+\mathrm{H}]^{+}, \mathrm{HRMS}(\mathrm{ESI})$ calcd. for $\mathrm{C}_{28} \mathrm{H}_{36} \mathrm{FN}_{2} \mathrm{O}_{5} \mathrm{~S}^{+}:[\mathrm{M}+\mathrm{H}]^{+}$ $m / z: 531.2323$, found: 531.2314 .

5-(2-Cyclopropyl-1-(2-fluorophenyl)-2-oxoethyl)-4,5,6,7-tetrahydrothieno[3,2-c]pyridin-2-yl-2-((tert-butoxycarbonyl)amino)-4-methylpentanoate (4k). Yellow oil. ${ }^{1} \mathrm{H}-\mathrm{NMR}\left(400 \mathrm{MHz}, \mathrm{DMSO}-d_{6}\right): 7.52-7.48(\mathrm{~m}, 2 \mathrm{H})$, $7.40(\mathrm{dd}, 1 \mathrm{H}, J=13.6 \mathrm{~Hz}), 7.26-7.21(\mathrm{~m}, 2 \mathrm{H}), 6.43(\mathrm{~s}, 1 \mathrm{H}), 4.78(\mathrm{~s}, 1 \mathrm{H}), 4.17-4.12(\mathrm{~m}, 1 \mathrm{H}), 3.44(\mathrm{t}, 2 \mathrm{H}$, $J=6.4 \mathrm{~Hz}), 2.80-2.70(\mathrm{~m}, 4 \mathrm{H}), 2.37-2.35(\mathrm{~m}, 1 \mathrm{H}), 1.66-1.42(\mathrm{~m}, 3 \mathrm{H}), 1.37(\mathrm{~s}, 9 \mathrm{H}), 0.90-0.80(\mathrm{~m}, 10 \mathrm{H})$. $\operatorname{ESI-MS}(m / z)=545.14[\mathrm{M}+\mathrm{H}]^{+}$.

5-(2-Cyclopropyl-1-(2-fluorophenyl)-2-oxoethyl)-4,5,6,7-tetrahydrothieno[3,2-c]pyridin-2-yl-2-((tert-butoxy-car bonyl)amino)-3-methylpentanoate (41). White solid. m.p.: 80-82 ${ }^{\circ} \mathrm{C}$. ${ }^{1} \mathrm{H}-\mathrm{NMR}\left(400 \mathrm{MHz}, \mathrm{DMSO}-d_{6}\right)$ : $\delta 7.52-7.45(\mathrm{~m}, 2 \mathrm{H}), 7.40(\mathrm{dd}, 1 \mathrm{H}, J=12.8 \mathrm{~Hz}), 7.26-7.21(\mathrm{~m}, 2 \mathrm{H}), 6.43(\mathrm{~s}, 1 \mathrm{H}), 4.77(\mathrm{~s}, 1 \mathrm{H}), 4.05(\mathrm{t}, 1 \mathrm{H}$, $J=6.8 \mathrm{~Hz}), 3.44(\mathrm{t}, 2 \mathrm{H}, J=15.2 \mathrm{~Hz}), 2.80-2.69(\mathrm{~m}, 4 \mathrm{H}), 2.39-2.35(\mathrm{~m}, 1 \mathrm{H}), 1.86-1.82(\mathrm{~m}, 1 \mathrm{H}), 1.38-1.20$ $(\mathrm{m}, 11 \mathrm{H}), 0.88-0.73(\mathrm{~m}, 10 \mathrm{H})$. ESI-MS $(m / z)=545.10[\mathrm{M}+\mathrm{H}]^{+}, \mathrm{HRMS}(\mathrm{ESI})$ calcd. for $\mathrm{C}_{29} \mathrm{H}_{38} \mathrm{FN}_{2} \mathrm{O}_{5} \mathrm{~S}^{+}$: $[\mathrm{M}+\mathrm{H}]^{+} m / z: 545.2480$, found: 545.2467 .

5-(2-Cyclopropyl-1-(2-fluorophenyl)-2-oxoethyl)-4,5,6,7-tetrahydrothieno[3,2-c]pyridin-2-yl-2-((tert-butoxy-car bonyl)amino)-3-phenylpropanoate (4m). White solid. m.p.: $110-112{ }^{\circ} \mathrm{C} .{ }^{1} \mathrm{H}-\mathrm{NMR}\left(400 \mathrm{MHz}, \mathrm{DMSO}-d_{6}\right)$ : $\delta 7.54(\mathrm{~d}, 1 \mathrm{H}, J=7.2 \mathrm{~Hz}), 7.48(\mathrm{t}, 1 \mathrm{H}, J=7.2 \mathrm{~Hz}), 7.40-7.36(\mathrm{~m}, 1 \mathrm{H}), 7.28-7.20(\mathrm{~m}, 7 \mathrm{H}), 6.32(\mathrm{~s}, 1 \mathrm{H}), 4.76$ $(\mathrm{s}, 1 \mathrm{H}), 4.35-4.14(\mathrm{~m}, 1 \mathrm{H}), 3.45-3.36(\mathrm{~m}, 2 \mathrm{H}), 3.05-3.00(\mathrm{~m}, 2 \mathrm{H}), 2.79-2.66(\mathrm{~m}, 4 \mathrm{H}), 2.36-2.34(\mathrm{~m}, 1 \mathrm{H})$, $1.31(\mathrm{~s}, 9 \mathrm{H}), 0.87-0.76(\mathrm{~m}, 4 \mathrm{H})$. ESI-MS $(\mathrm{m} / z)=579.17[\mathrm{M}+\mathrm{H}]^{+}, \mathrm{HRMS}(\mathrm{ESI})$ calcd. for $\mathrm{C}_{32} \mathrm{H}_{36} \mathrm{FN}_{2} \mathrm{O}_{5} \mathrm{~S}^{+}$: $[\mathrm{M}+\mathrm{H}]^{+} m / z: 579.2323$, found: 579.2315 . 
5-(2-Cyclopropyl-1-(2-fluorophenyl)-2-oxoethyl)-4,5,6,7-tetrahydrothieno[3,2-c]pyridin-2-yl-2-((tertbutoxycarbonyl)amino)-3-(1H-indol-2-yl)propanoate (4n). Yellow oil. ${ }^{1} \mathrm{H}-\mathrm{NMR}\left(400 \mathrm{MHz}, \mathrm{DMSO}-d_{6}\right): \delta 10.86$ (s, $1 \mathrm{H}), 7.52-7.49(\mathrm{~m}, 3 \mathrm{H}), 7.40(\mathrm{dd}, 1 \mathrm{H}, J=13.2 \mathrm{~Hz}), 7.33(\mathrm{~d}, 1 \mathrm{H}, J=8.0 \mathrm{~Hz}), 7.27-7.22(\mathrm{~m}, 2 \mathrm{H}), 7.18(\mathrm{~s}, 1 \mathrm{H})$, 7.08-6.95 (m, 2H), $6.26(\mathrm{~s}, 1 \mathrm{H}), 4.78(\mathrm{~s}, 1 \mathrm{H}), 4.40-4.36(\mathrm{~m}, 1 \mathrm{H}), 3.45-3.37(\mathrm{~m}, 2 \mathrm{H}), 3.18-3.14(\mathrm{~m}, 2 \mathrm{H})$, 2.80-2.68 (m, 4H), 2.38-2.36 (m, 1H), $1.34(\mathrm{~s}, 9 \mathrm{H}), 0.88-0.80(\mathrm{~m}, 4 \mathrm{H})$. ESI-MS $(\mathrm{m} / \mathrm{z})=618.23[\mathrm{M}+\mathrm{H}]^{+}$.

1-tert-Butyl 2-(5-(2-cyclopropyl-1-(2-fluorophenyl)-2-oxoethyl)-4,5,6,7-tetrahydrothieno[3,2-c]pyridin-2-yl) pyrrolidine-1,2-dicarboxylate (4o). Yellow oil. ${ }^{1} \mathrm{H}-\mathrm{NMR}\left(400 \mathrm{MHz}, \mathrm{DMSO}-d_{6}\right): \delta 7.50,(\mathrm{t}, 1 \mathrm{H}, J=7.2 \mathrm{~Hz})$, $7.40(\mathrm{dd}, 1 \mathrm{H}, J=13.6 \mathrm{~Hz}), 7.28-7.22(\mathrm{~m}, 2 \mathrm{H}), 6.47(\mathrm{~s}, 1 \mathrm{H}), 4.78(\mathrm{~s}, 1 \mathrm{H}), 4.42-4.36(\mathrm{~m}, 1 \mathrm{H}), 3.65(\mathrm{~m}, 1 \mathrm{H})$, $3.48-3.33(\mathrm{~m}, 4 \mathrm{H}), 3.03-2.79(\mathrm{~m}, 4 \mathrm{H}), 2.37-2.24(\mathrm{~m}, 2 \mathrm{H}), 2.02-2.00(\mathrm{~m}, 1 \mathrm{H}), 1.98-1.87(\mathrm{~m}, 2 \mathrm{H}), 1.39(\mathrm{~s}$, 9H), 0.88-0.75 $(\mathrm{m}, 4 \mathrm{H})$. ESI-MS $(m / z)=529.10[\mathrm{M}+\mathrm{H}]^{+}$.

1-tert-Butyl 2-(5-(2-cyclopropyl-1-(2-fluorophenyl)-2-oxoethyl)-4,5,6,7-tetrahydrothieno[3,2-c]pyridin-2-yl) 5-oxopyrrolidine-1,2-dicarboxylate (4p). Yellow oil. ${ }^{1} \mathrm{H}-\mathrm{NMR}\left(400 \mathrm{MHz}, \mathrm{DMSO}-d_{6}\right): \delta 7.50(\mathrm{t}, 1 \mathrm{H}$, $J=7.2 \mathrm{~Hz}), 7.40(\mathrm{dd}, 1 \mathrm{H}, J=13.2 \mathrm{~Hz}), 7.27-7.22(\mathrm{~m}, 2 \mathrm{H}), 6.53(\mathrm{~s}, 1 \mathrm{H}), 4.87(\mathrm{q}, 1 \mathrm{H}, J=5.6 \mathrm{HZ}), 4.79(\mathrm{~s}, 1 \mathrm{H})$, 3.49-3.41 (m, 2H), 2.82-2.69 (m, 4H), 2.56-2.34 (m, 4H), 2.08-2.01 (m, 1H), $1.39(\mathrm{~s}, 9 \mathrm{H}), 0.88-0.84(\mathrm{~m}$, $4 \mathrm{H})$. ESI-MS $(m / z)=543.15[\mathrm{M}+\mathrm{H}]^{+}$.

5-(1-(2-Chlorophenyl)-2-methoxy-2-oxoethyl)-4,5,6,7-tetrahydrothieno[3,2-c]pyridin-2-yl 2-aminopropanoate hydrochloride (5a). Compound $4 \mathbf{a}(1.0 \mathrm{~g}, 2.0 \mathrm{mmol})$ was stirred with hydrochloric ethyl acetate $(10 \mathrm{~mL}$, $2.0 \mathrm{M})$ at r.t. for $5.0 \mathrm{~h}$. The formed precipitate was filtered and dried to give compound $5 \mathbf{a}(0.82 \mathrm{~g}$, 92.1\%) as a white solid. m.p.: $149-151{ }^{\circ} \mathrm{C} .{ }^{1} \mathrm{H}-\mathrm{NMR}\left(400 \mathrm{MHz}, \mathrm{DMSO}-d_{6}\right): \delta 8.80(\mathrm{~s}, 3 \mathrm{H}), 7.57-7.55(\mathrm{~m}$, $1 \mathrm{H}), 7.52-7.40(\mathrm{~m}, 3 \mathrm{H}), 6.62(\mathrm{~s}, 1 \mathrm{H}), 5.30(\mathrm{~m}, 1 \mathrm{H}), 4.38(\mathrm{~s}, 1 \mathrm{H}), 3.93(\mathrm{~s}, 2 \mathrm{H}), 3.70(\mathrm{~s}, 3 \mathrm{H}), 3.20-2.88(\mathrm{~m}$, $4 \mathrm{H}), 1.52(\mathrm{~d}, 3 \mathrm{H}, J=8.0 \mathrm{~Hz})$. ESI-MS $(\mathrm{m} / z)=408.98[\mathrm{M}+\mathrm{H}]^{+}$, HRMS(ESI) calcd. for $\mathrm{C}_{19} \mathrm{H}_{22} \mathrm{ClN}_{2} \mathrm{O}_{4} \mathrm{~S}^{+}$: $[\mathrm{M}+\mathrm{H}]^{+} \mathrm{m} / z: 409.0983$, found: 409.0986 .

Compounds $5 \mathbf{b}-\mathbf{5} \mathbf{p}$ were synthesized according to the procedure described for the preparation of $5 a$.

5-(1-(2-Chlorophenyl)-2-methoxy-2-oxoethyl)-4,5,6,7-tetrahydrothieno[3,2-c]pyridin-2-yl-2-amino-3-methylbutanoate hydrochloride (5b). White solid. m.p.: $165-167{ }^{\circ} \mathrm{C} .{ }^{1} \mathrm{H}-\mathrm{NMR}\left(400 \mathrm{MHz}, \mathrm{DMSO}-d_{6}\right): \delta 8.93$ (s, $3 \mathrm{H}), 7.77(\mathrm{~s}, 1 \mathrm{H}), 7.63-7.58(\mathrm{~m}, 1 \mathrm{H}), 7.52-7.46(\mathrm{~m}, 2 \mathrm{H}), 6.64(\mathrm{~s}, 1 \mathrm{H}), 5.34(\mathrm{~m}, 1 \mathrm{H}), 4.15(\mathrm{~s}, 1 \mathrm{H}), 3.93(\mathrm{~s}$, 2H), $3.70(\mathrm{~s}, 3 \mathrm{H}), 3.23-2.92(\mathrm{~m}, 4 \mathrm{H}), 2.34-2.29(\mathrm{~m}, 1 \mathrm{H}), 1.05-0.98(\mathrm{~m}, 6 \mathrm{H})$. ESI-MS $(\mathrm{m} / z)=437.02[\mathrm{M}+$ $\mathrm{H}]^{+}, \mathrm{HRMS}(\mathrm{ESI})$ calcd. for $\mathrm{C}_{21} \mathrm{H}_{26} \mathrm{ClN}_{2} \mathrm{O}_{4} \mathrm{~S}^{+}:[\mathrm{M}+\mathrm{H}]^{+} m / z$ : 437.1296, found: 437.1333.

5-(1-(2-Chlorophenyl)-2-methoxy-2-oxoethyl)-4,5,6,7-tetrahydrothieno[3,2-c]pyridin-2-yl-2-amino-4-methylpentanoate hydrochloride (5c). White solid. m.p.: $133-135^{\circ} \mathrm{C} .{ }^{1} \mathrm{H}-\mathrm{NMR}\left(400 \mathrm{MHz}, \mathrm{DMSO}-d_{6}\right): \delta 8.82(\mathrm{~s}$, $3 \mathrm{H}), 7.69-7.64(\mathrm{~m}, 1 \mathrm{H}), 7.62-7.59(\mathrm{~m}, 1 \mathrm{H}), 7.51-7.40(\mathrm{~m}, 2 \mathrm{H}), 6.62(\mathrm{~s}, 1 \mathrm{H}), 5.20(\mathrm{~m}, 1 \mathrm{H}), 4.26(\mathrm{~s}, 1 \mathrm{H})$, $3.89(\mathrm{~s}, 2 \mathrm{H}), 3.70(\mathrm{~s}, 3 \mathrm{H}), 3.12-2.86(\mathrm{~m}, 4 \mathrm{H}), 2.70-2.66(\mathrm{~m}, 2 \mathrm{H}), 1.63-1.59(\mathrm{~m}, 1 \mathrm{H}), 0.92-0.90(\mathrm{~m}, 6 \mathrm{H})$. ESI-MS $(m / z)=451.03[\mathrm{M}+\mathrm{H}]^{+}, \mathrm{HRMS}(\mathrm{ESI})$ calcd. for $\mathrm{C}_{22} \mathrm{H}_{28} \mathrm{ClN}_{2} \mathrm{O}_{4} \mathrm{~S}^{+}:[\mathrm{M}+\mathrm{H}]^{+} m / z: 451.1453$, found: 451.1455 . HPLC purity: $94.00 \%$.

5-(1-(2-Chlorophenyl)-2-methoxy-2-oxoethyl)-4,5,6,7-tetrahydrothieno[3,2-c]pyridin-2-yl-2-amino-3-methylpentanoate hydrochloride (5d). White solid. m.p.: $150-153{ }^{\circ} \mathrm{C} .{ }^{1} \mathrm{H}-\mathrm{NMR}\left(400 \mathrm{MHz}, \mathrm{DMSO}-d_{6}\right): \delta 8.91$ (s, $3 \mathrm{H}), 7.75-7.72(\mathrm{~m}, 1 \mathrm{H}), 7.64-7.62(\mathrm{~m}, 1 \mathrm{H}), 7.56-7.46(\mathrm{~m}, 2 \mathrm{H}), 6.63(\mathrm{~s}, 1 \mathrm{H}), 5.30-5.26(\mathrm{~m}, 1 \mathrm{H}), 4.21(\mathrm{~s}, 1 \mathrm{H})$, $3.87(\mathrm{~s}, 2 \mathrm{H}), 3.70(\mathrm{~s}, 3 \mathrm{H}), 3.17-2.88(\mathrm{~m}, 4 \mathrm{H}), 2.08-1.99(\mathrm{~m}, 1 \mathrm{H}), 1.56-1.49(\mathrm{~m}, 1 \mathrm{H}), 1.36-1.29(\mathrm{~m}, 1 \mathrm{H})$, 0.94-0.87 $(\mathrm{m}, 6 \mathrm{H})$. ESI-MS $(m / z)=451.04[\mathrm{M}+\mathrm{H}]^{+}$, HRMS(ESI) calcd. for $\mathrm{C}_{22} \mathrm{H}_{28} \mathrm{ClN}_{2} \mathrm{O}_{4} \mathrm{~S}^{+}:[\mathrm{M}+\mathrm{H}]^{+}$ $m / z: 451.1453$, found: 451.1459 .

5-(1-(2-Chlorophenyl)-2-methoxy-2-oxoethyl)-4,5,6,7-tetrahydrothieno[3,2-c]pyridin-2-yl-2-amino-3-phenylpropanoate hydrochloride (5e). White solid. m.p.: $166-168^{\circ} \mathrm{C} .{ }^{1} \mathrm{H}-\mathrm{NMR}\left(400 \mathrm{MHz}, \mathrm{DMSO}-d_{6}\right): \delta 9.01$ (s, $3 \mathrm{H}), 7.65-7.57(\mathrm{~m}, 3 \mathrm{H}), 7.46(\mathrm{~d}, 2 \mathrm{H}, J=6.4 \mathrm{~Hz}), 7.33-7.27(\mathrm{~m}, 4 \mathrm{H}), 6.43(\mathrm{~s}, 1 \mathrm{H}), 5.30-5.25(\mathrm{~m}, 1 \mathrm{H}), 4.54(\mathrm{~s}$, $1 \mathrm{H}), 3.89(\mathrm{~s}, 2 \mathrm{H}), 3.70(\mathrm{~s}, 3 \mathrm{H}), 3.39-3.16(\mathrm{~m}, 2 \mathrm{H}), 3.14-2.87(\mathrm{~m}, 4 \mathrm{H})$. ESI-MS $(\mathrm{m} / \mathrm{z})=485.03[\mathrm{M}+\mathrm{H}]^{+}$, HRMS(ESI) calcd. for $\mathrm{C}_{25} \mathrm{H}_{26} \mathrm{ClN}_{2} \mathrm{O}_{4} \mathrm{~S}^{+}:[\mathrm{M}+\mathrm{H}]^{+} m / z$ : 485.1296, found: 485.1296 . 
5-(1-(2-Chlorophenyl)-2-methoxy-2-oxoethyl)-4,5,6,7-tetrahydrothieno[3,2-c]pyridin-2-yl-2-amino-3-(1H-indol2-yl)propanoate hydrochloride (5f). White solid. m.p.: $173-175{ }^{\circ} \mathrm{C} .{ }^{1} \mathrm{H}-\mathrm{NMR}\left(400 \mathrm{MHz}\right.$, DMSO- $\left.d_{6}\right)$ : $\delta 11.10(\mathrm{~s}, 1 \mathrm{H}), 8.92(\mathrm{~s}, 3 \mathrm{H}), 7.57(\mathrm{~d}, 2 \mathrm{H}, J=7.2 \mathrm{~Hz}), 7.52-7.50(\mathrm{~m}, 3 \mathrm{H}), 7.38(\mathrm{~d}, 1 \mathrm{H}, J=6.4 \mathrm{~Hz}), 7.25(\mathrm{~s}$, $1 \mathrm{H}), 7.08(\mathrm{t}, 1 \mathrm{H}, J=12.0 \mathrm{~Hz}), 6.98(\mathrm{t}, 1 \mathrm{H}, J=16.0 \mathrm{~Hz}), 6.36(\mathrm{~s}, 1 \mathrm{H}), 5.28-5.24(\mathrm{~m}, 1 \mathrm{H}), 4.50(\mathrm{~s}, 1 \mathrm{H}), 3.93(\mathrm{~s}$, $2 \mathrm{H}), 3.70(\mathrm{~s}, 3 \mathrm{H}), 3.51-3.46(\mathrm{~m}, 1 \mathrm{H}), 3.38-3.33(\mathrm{~m}, 1 \mathrm{H}), 3.20-2.88(\mathrm{~m}, 4 \mathrm{H})$. ESI-MS $(\mathrm{m} / z)=524.09[\mathrm{M}+$ $\mathrm{H}]^{+}, \mathrm{HRMS}(\mathrm{ESI})$ calcd. for $\mathrm{C}_{27} \mathrm{H}_{27} \mathrm{ClN}_{3} \mathrm{O}_{4} \mathrm{~S}^{+}:[\mathrm{M}+\mathrm{H}]^{+} \mathrm{m} / z: 524.1405$, found: 524.1408 .

5-(1-(2-Chlorophenyl)-2-methoxy-2-oxoethyl)-4,5,6,7-tetrahydrothieno[3,2-c]pyridin-2-yl-pyrrolidine-2-car boxylate hydrochloride (5g). White solid. m.p.: 102-104 ${ }^{\circ} \mathrm{C} .{ }^{1} \mathrm{H}-\mathrm{NMR}\left(400 \mathrm{MHz}\right.$, DMSO- $\left.d_{6}\right): \delta 10.47$ $(\mathrm{s}, 1 \mathrm{H}), 9.47(\mathrm{~s}, 1 \mathrm{H}), 7.72(\mathrm{~d}, 1 \mathrm{H}, J=8.8 \mathrm{~Hz}), 7.62-7.52(\mathrm{~m}, 1 \mathrm{H}), 7.41-7.39(\mathrm{~m}, 2 \mathrm{H}), 6.63(\mathrm{~s}, 1 \mathrm{H})$, 5.29-5.24 (m, 1H), $4.64(\mathrm{~s}, 1 \mathrm{H}), 3.88(\mathrm{~s}, 2 \mathrm{H}), 3.70(\mathrm{~s}, 3 \mathrm{H}), 3.23-3.20(\mathrm{~m}, 4 \mathrm{H}), 2.90-2.88(\mathrm{~m}, 2 \mathrm{H}), 2.38-2.29$ $(\mathrm{m}, 1 \mathrm{H}), 2.17-2.10(\mathrm{~m}, 1 \mathrm{H}), 1.97-1.90(\mathrm{~m}, 2 \mathrm{H})$. ESI-MS $(\mathrm{m} / \mathrm{z})=434.96[\mathrm{M}+\mathrm{H}]^{+}$, HRMS(ESI) calcd. for $\mathrm{C}_{21} \mathrm{H}_{24} \mathrm{ClN}_{2} \mathrm{O}_{4} \mathrm{~S}^{+}:[\mathrm{M}+\mathrm{H}]^{+} m / z: 435.1140$, found: 435.1147 .

5-(1-(2-Chlorophenyl)-2-methoxy-2-oxoethyl)-4,5,6,7-tetrahydrothieno[3,2-c]pyridin-2-yl-5-oxopyrrolidine-2-car boxylate hydrochloride (5h). White solid. m.p.: $88-90{ }^{\circ} \mathrm{C} .{ }^{1} \mathrm{H}-\mathrm{NMR}\left(400 \mathrm{MHz}, \mathrm{DMSO}-d_{6}\right): \delta 8.10$ (s, $1 \mathrm{H}), 7.76(\mathrm{~s}, 1 \mathrm{H}), 7.61-7.59(\mathrm{~m}, 1 \mathrm{H}), 7.51-7.39(\mathrm{~m}, 3 \mathrm{H}), 6.59(\mathrm{~s}, 1 \mathrm{H}), 5.42-5.37(\mathrm{~m}, 1 \mathrm{H}), 4.49-4.46(\mathrm{~m}$, $1 \mathrm{H}), 3.71(\mathrm{~s}, 3 \mathrm{H}), 3.67(\mathrm{~s}, 2 \mathrm{H}), 3.04-2.90(\mathrm{~m}, 4 \mathrm{H}), 2.47-2.10(\mathrm{~m}, 4 \mathrm{H})$. ESI-MS $(\mathrm{m} / \mathrm{z})=449.00[\mathrm{M}+\mathrm{H}]^{+}$, HRMS(ESI) calcd. for $\mathrm{C}_{21} \mathrm{H}_{22} \mathrm{ClN}_{2} \mathrm{O}_{5} \mathrm{~S}^{+}:[\mathrm{M}+\mathrm{H}]^{+} m / z: 449.0932$, found: 449.0932 .

5-(2-Cyclopropyl-1-(2-fluorophenyl)-2-oxoethyl)-4,5,6,7-tetrahydrothieno[3,2-c]pyridin-2-yl-2-amino-propanoate hydrochloride (5i). White solid. m.p.: $148-150{ }^{\circ} \mathrm{C} .{ }^{1} \mathrm{H}-\mathrm{NMR}\left(400 \mathrm{MHz}, \mathrm{DMSO}-d_{6}\right): \delta 8.90(\mathrm{~s}, 3 \mathrm{H})$, 7.66-7.55 (m, 2H), 7.50-7.33 (m, 2H), $6.67(\mathrm{~s}, 1 \mathrm{H}), 6.08-6.03(\mathrm{~m}, 1 \mathrm{H}), 4.38(\mathrm{~s}, 1 \mathrm{H}), 3.99(\mathrm{~s}, 2 \mathrm{H}), 3.61-3.57$ $(\mathrm{m}, 2 \mathrm{H}), 3.10-2.91(\mathrm{~m}, 2 \mathrm{H}), 1.98-1.89(\mathrm{~m}, 1 \mathrm{H}), 1.50(\mathrm{~d}, 3 \mathrm{H}, J=17.2 \mathrm{~Hz}), 1.07-0.93(\mathrm{~m}, 4 \mathrm{H})$. ESI-MS $(\mathrm{m} / \mathrm{z})$ $=403.05[\mathrm{M}+\mathrm{H}]^{+}, \mathrm{HRMS}(\mathrm{ESI})$ calcd. for $\mathrm{C}_{21} \mathrm{H}_{24} \mathrm{FN}_{2} \mathrm{O}_{3} \mathrm{~S}^{+}:[\mathrm{M}+\mathrm{H}]^{+} m / z: 403.1486$, found: 403.1491 .

5-(2-Cyclopropyl-1-(2-fluorophenyl)-2-oxoethyl)-4,5,6,7-tetrahydrothieno[3,2-c]pyridin-2-yl-2-amino-3-methyl butanoate hydrochloride (5j). White solid. m.p.: $147-148{ }^{\circ} \mathrm{C} .{ }^{1} \mathrm{H}-\mathrm{NMR}\left(400 \mathrm{MHz}, \mathrm{DMSO}-d_{6}\right): \delta 8.90$ (s, $3 \mathrm{H}), 7.62(\mathrm{~d}, 2 \mathrm{H}, J=6.4 \mathrm{~Hz}), 7.45-7.36(\mathrm{~m}, 2 \mathrm{H}), 6.69(\mathrm{~s}, 1 \mathrm{H}), 6.01-5.97(\mathrm{~m}, 1 \mathrm{H}), 4.16(\mathrm{~s}, 1 \mathrm{H}), 3.96(\mathrm{~s}$, $2 \mathrm{H}), 3.65-3.49(\mathrm{~m}, 2 \mathrm{H}), 3.04-3.00(\mathrm{~m}, 2 \mathrm{H}), 2.34-2.26(\mathrm{~m}, 1 \mathrm{H}), 1.97-1.93(\mathrm{~m}, 1 \mathrm{H}), 1.16-0.90(\mathrm{~m}, 10 \mathrm{H})$. ESI-MS $(m / z)=431.09[\mathrm{M}+\mathrm{H}]^{+}$, HRMS(ESI) calcd. for $\mathrm{C}_{23} \mathrm{H}_{28} \mathrm{FN}_{2} \mathrm{O}_{3} \mathrm{~S}^{+}:[\mathrm{M}+\mathrm{H}]^{+} m / z: 431.1799$, found: 431.1798 .

5-(2-Cyclopropyl-1-(2-fluorophenyl)-2-oxoethyl)-4,5,6,7-tetrahydrothieno[3,2-c]pyridin-2-yl-2-amino-4-methyl pentanoate hydrochloride (5k). White solid. m.p.: $141-143{ }^{\circ} \mathrm{C} .{ }^{1} \mathrm{H}-\mathrm{NMR}\left(400 \mathrm{MHz}, \mathrm{DMSO}-d_{6}\right): \delta 8.64$ $(\mathrm{s}, 3 \mathrm{H}), 7.70-7.62(\mathrm{~m}, 2 \mathrm{H}), 7.47-7.37(\mathrm{~m}, 2 \mathrm{H}), 6.68(\mathrm{~s}, 1 \mathrm{H}), 6.08-6.01(\mathrm{~m}, 1 \mathrm{H}), 4.23(\mathrm{~s}, 1 \mathrm{H}), 3.98(\mathrm{~s}, 2 \mathrm{H})$, 3.61-3.48 (m, 2H), 3.05-3.00 (m, 2H), 2.76-2.71 (m, 2H), 1.97-1.87 (m, 1H), 1.65-1.63 (m, 1H), 0.91-0.90 $(\mathrm{m}, 10 \mathrm{H})$. ESI-MS $(m / z)=445.08[\mathrm{M}+\mathrm{H}]^{+}, \mathrm{HRMS}(\mathrm{ESI})$ calcd. for $\mathrm{C}_{24} \mathrm{H}_{30} \mathrm{FN}_{2} \mathrm{O}_{3} \mathrm{~S}^{+}:[\mathrm{M}+\mathrm{H}]^{+} \mathrm{m} / z$ : 445.1956, found: 445.1958 .

5-(2-Cyclopropyl-1-(2-fluorophenyl)-2-oxoethyl)-4,5,6,7-tetrahydrothieno[3,2-c]pyridin-2-yl-2-amino-3-methyl pentanoate hydrochloride (51). White solid. m.p.: $142-144{ }^{\circ} \mathrm{C} .{ }^{1} \mathrm{H}-\mathrm{NMR}\left(400 \mathrm{MHz}, \mathrm{DMSO}-d_{6}\right): \delta 8.90$ (s, 3H), 7.62-7.60 (m, 2H), 7.45-7.36 (m, 2H), $6.68(\mathrm{~s}, 1 \mathrm{H}), 6.08-6.03(\mathrm{~m}, 1 \mathrm{H}), 4.22(\mathrm{~s}, 1 \mathrm{H}), 3.85(\mathrm{~s}$, $2 \mathrm{H}), 3.15-2.92(\mathrm{~m}, 4 \mathrm{H}), 2.06-1.88(\mathrm{~m}, 2 \mathrm{H}), 1.54-1.49(\mathrm{~m}, 1 \mathrm{H}), 1.36-1.32(\mathrm{~m}, 1 \mathrm{H}), 1.03-0.87(\mathrm{~m}, 10 \mathrm{H})$. ESI-MS $(m / z)=445.07[\mathrm{M}+\mathrm{H}]^{+}$, HRMS(ESI) calcd. for $\mathrm{C}_{24} \mathrm{H}_{30} \mathrm{FN}_{2} \mathrm{O}_{3} \mathrm{~S}^{+}:[\mathrm{M}+\mathrm{H}]^{+} m / z: 445.1956$, found: 445.1957 .

5-(2-Cyclopropyl-1-(2-fluorophenyl)-2-oxoethyl)-4,5,6,7-tetrahydrothieno[3,2-c]pyridin-2-yl-2-amino-3-phenyl propanoate hydrochloride $(\mathbf{5 m})$. White solid. m.p.: $156-158{ }^{\circ} \mathrm{C} .{ }^{1} \mathrm{H}-\mathrm{NMR}\left(400 \mathrm{MHz}, \mathrm{DMSO}-d_{6}\right): \delta 9.06(\mathrm{~s}$, $3 \mathrm{H}), 7.65-7.63(\mathrm{~m}, 2 \mathrm{H}), 7.43-7.28(\mathrm{~m}, 7 \mathrm{H}), 6.48(\mathrm{~s}, 1 \mathrm{H}), 6.06-6.02(\mathrm{~m}, 1 \mathrm{H}), 4.53(\mathrm{~s}, 1 \mathrm{H}), 3.88(\mathrm{~s}, 2 \mathrm{H})$, 3.39-3.15 (m, 2H), 3.14-2.87 (m, 4H), 1.99-1.95 (m, 1H), 0.91-0.89 (m, 4H). ESI-MS $(m / z)=479.05[\mathrm{M}+$ $\mathrm{H}]^{+}, \mathrm{HRMS}(\mathrm{ESI})$ calcd. for $\mathrm{C}_{27} \mathrm{H}_{28} \mathrm{FN}_{2} \mathrm{O}_{3} \mathrm{~S}^{+}:[\mathrm{M}+\mathrm{H}]^{+} m / z: 479.1799$, found: 479.1798 .

5-(2-Cyclopropyl-1-(2-fluorophenyl)-2-oxoethyl)-4,5,6,7-tetrahydrothieno[3,2-c]pyridin-2-yl-2-amino-3-(1Hindol-2-yl)propanoate hydrochloride (5n). White solid. m.p.: $166-168{ }^{\circ} \mathrm{C} .{ }^{1} \mathrm{H}-\mathrm{NMR}(400 \mathrm{MHz}, \mathrm{DMSO}-\mathrm{d} 6)$ : 
$\delta 11.11(\mathrm{~s}, 1 \mathrm{H}), 8.93(\mathrm{~s}, 3 \mathrm{H}), 7.61-7.56(\mathrm{~m}, 3 \mathrm{H}), 7.46-7.30(\mathrm{~m}, 3 \mathrm{H}), 7.25(\mathrm{~s}, 1 \mathrm{H}), 7.07(\mathrm{t}, 1 \mathrm{H}, \mathrm{J}=7.6 \mathrm{~Hz})$, $6.99(\mathrm{t}, 1 \mathrm{H}, J=7.2 \mathrm{~Hz}), 6.43(\mathrm{~s}, 1 \mathrm{H}), 4.97-4.95(\mathrm{~m}, 1 \mathrm{H}), 4.50(\mathrm{~s}, 1 \mathrm{H}), 4.00(\mathrm{~s}, 2 \mathrm{H}), 3.51-3.23(\mathrm{~m}, 2 \mathrm{H})$, 3.20-2.88 (m, 4H), 1.91-1.89 (m, 1H), 1.04-0.89 (m, 4H). ESI-MS $(m / z)=518.09[\mathrm{M}+\mathrm{H}]^{+}, \mathrm{HRMS}(\mathrm{ESI})$ calcd. for $\mathrm{C}_{29} \mathrm{H}_{29} \mathrm{FN}_{3} \mathrm{O}_{3} \mathrm{~S}^{+}:[\mathrm{M}+\mathrm{H}]^{+} m / z: 518.1908$, found: 518.1912 .

5-(2-Cyclopropyl-1-(2-fluorophenyl)-2-oxoethyl)-4,5,6,7-tetrahydrothieno[3,2-c]pyridin-2-yl-pyrrolidine-2-car boxylate hydrochloride (5o). White solid. m.p.: $96-98{ }^{\circ} \mathrm{C} .{ }^{1} \mathrm{H}-\mathrm{NMR}\left(400 \mathrm{MHz}, \mathrm{DMSO}-d_{6}\right): \delta 10.74(\mathrm{~s}, 1 \mathrm{H})$, $9.72(\mathrm{~s}, 1 \mathrm{H}), 7.69-7.62(\mathrm{~m}, 2 \mathrm{H}), 7.47-7.37(\mathrm{~m}, 2 \mathrm{H}), 6.69(\mathrm{~s}, 1 \mathrm{H}), 6.10-6.03(\mathrm{~m}, 1 \mathrm{H}), 4.63(\mathrm{~s}, 1 \mathrm{H}), 4.23-4.19$ (s, 2H), 3.57-3.47 (m, 2H), 3.28-3.06 (m, 4H), 2.34-2.10 (m,2H), 1.97-1.90 (m, 3H), 1.07-0.90 (m, 4H). ESI-MS $(m / z)=429.08[\mathrm{M}+\mathrm{H}]^{+}, \mathrm{HRMS}(\mathrm{ESI})$ calcd. for $\mathrm{C}_{23} \mathrm{H}_{26} \mathrm{FN}_{2} \mathrm{O}_{3} \mathrm{~S}^{+}:[\mathrm{M}+\mathrm{H}]^{+} m / z: 429.1643$, found: 429.1640 .

5-(2-Cyclopropyl-1-(2-fluorophenyl)-2-oxoethyl)-4,5,6,7-tetrahydrothieno[3,2-c]pyridin-2-yl-5-oxo-pyrrolidine2-carboxylate hydrochloride (5p). White solid. m.p.: $98-101{ }^{\circ} \mathrm{C} .{ }^{1} \mathrm{H}-\mathrm{NMR}\left(400 \mathrm{MHz}, \mathrm{DMSO}-d_{6}\right): \delta 8.11(\mathrm{~s}$, $1 \mathrm{H}), 7.64-7.39(\mathrm{~m}, 5 \mathrm{H}), 6.63(\mathrm{~s}, 1 \mathrm{H}), 6.09-6.03(\mathrm{~m}, 1 \mathrm{H}), 4.48-4.47(\mathrm{~m}, 1 \mathrm{H}), 3.44(\mathrm{~s}, 2 \mathrm{H}), 3.04(\mathrm{~m}, 2 \mathrm{H})$, 2.44-2.40 (m, 1H), 2.20-2.12 (m, 3H), 1.97-1.90 (m, 3H), 1.05-0.90 (m, 4H). ESI-MS $(m / z)=443.06[\mathrm{M}+$ $\mathrm{H}]^{+}, \mathrm{HRMS}(\mathrm{ESI})$ calcd. for $\mathrm{C}_{23} \mathrm{H}_{24} \mathrm{FN}_{2} \mathrm{O}_{4} \mathrm{~S}^{+}:[\mathrm{M}+\mathrm{H}]^{+} m / z: 443.1435$, found: 443.1433 .

\subsection{Inhibition of ADP-Induced Platelet Aggregation in Rats}

ADP-induced platelet aggregation was determined by Born's method [28]. Male SD rats (200-300 g, 5 in each group) were orally gavaged at random with vehicle control, target compounds, clopidogrel and prasugrel. The volume of target compounds and positive control was $10 \mathrm{~mL} / \mathrm{kg}$ $\times$ body weight, while the vehicle group was equal volume as experimental group instead of $0.5 \%$ CMC-Na. Two hours after administration, the animals were anesthetized (with $0.7 \%$ chloral hydrate intraperitoneal injection) and bloods were collected via aorta ventralis puncture into anticoagulant solution (3.8\% sodium citrate). Platelet rich plasma (PRP) was centrifuged at $230 \mathrm{rpm}$ for $15 \mathrm{mins}$ and then adjusted by platelet poor plasma (PPP, centrifuged at $2000 \mathrm{rpm}$ for $10 \mathrm{mins}$ ). Platelet count was $5 \times 10^{8} / \mathrm{mL}$. Aggregation was induced by ADP $(20 \mu \mathrm{M})$ and measured using an aggregometer (LBY-NJ4, Beijing, China ). The platelet aggregation was observed maximum platelet aggregation (MPA) from the aggregometer. The percentage of inhibition of platelet aggregation (IPA) was calculated from the observed MPA by the following equation:

$$
\operatorname{IPA}(\%)=\left(\mathrm{MPA}_{\text {vehicle }}-\mathrm{MPA}_{\text {compound }}\right) / \mathrm{MPA}_{\text {vehicle }} \times 100 \%
$$

Inhibition rats at different doses were calculated $\mathrm{ED}_{50}$ by the software named Statistical Product and Service Solutions (SPSS). The animal laboratory got animal use certificate issued by Science and Technology department of Tianjin (SYXK(jin)2016-0013).

\subsection{Determination of Bleeding Time}

The tail transection bleeding time was determined by the method of Dejana et al. [29]. Male SD rats (200-300 g, 5 in each group) were orally gavaged at random with vehicle control, target compounds, clopidogrel and prasugrel. The volume of target compounds and positive control was $10 \mathrm{~mL} / \mathrm{kg}$ $\times$ body weight, while the vehicle group was equal volume as experimental group instead of $0.5 \%$ CMC-Na. The test drugs were orally administered $1 \mathrm{H}$ before the tail transection. Under anaesthesia with urethane $(5 \mathrm{~mL} / \mathrm{kg})$, the rat tail was transected at $5 \mathrm{~mm}$ from the tip by a scalpel, and the tail was immediately immersed into warmed $\left(37^{\circ} \mathrm{C}\right)$ saline until blood flow stopped. Bleeding time was assessed as the time from the tail transection to the termination of blood flow. Bleeding times beyond $60 \mathrm{~min}$ were regarded as $60 \mathrm{~min}$ for the purpose of statistical analysis. $\mathrm{BT}_{2}$ values were calculated from linear-regression analysis. 


\section{Conclusions}

In summary, we designed and synthesized a series of amino acid prodrugs based on thienopyridine scaffolds as novel potent $\mathrm{P} 2 \mathrm{Y}_{12} \mathrm{R}$ inhibitors. Inhibition of ADP-induced platelet aggregation in rat assays showed that compounds $5 \mathbf{k}, \mathbf{5 1}, \mathbf{5 c}, \mathbf{5 j}, \mathbf{5 m}$, and $\mathbf{5 n}$ displayed good activity and compounds $5 \mathrm{c}$ and $\mathbf{5 l}$ have moderate $\mathrm{ED}_{50}$ values between those of clopidogrel and prasugrel. In the tail bleeding test, $5 \mathrm{c}$ induced much shorter bleeding time in rats and 51 behaved slightly better than prasugrel. Based on their ratio of benefit/bleeding risk, we will take $\mathbf{5 c}$ as a drug candidate for further research.

Author Contributions: Nan Lu and Lingjun Li conceived, designed and performed the synthetic experiment part; Yuquan Li and Jing Yuan analyzed the data; Xuemin Zheng, Shijun Zhang and Qunchao Wei conceived, designed and performed the pharmacological test part; Nan Lu wrote the paper. Youjun Xu and Fancui Meng assisted paper revision.

Acknowledgments: This project was supported by Tianjin Natural Science Foundation-Young Foundation (Nos. 17JCQNJC13700 and 17JCQNJC13100) and Innovative Research Team in SYPHU by the supporting Fund for Universities from the Chinese Central Government (51150039). And we also thank the innovative research team of the Ministry of Education and program for Liaoning innovative research team in university.

Conflicts of Interest: The authors declare no conflict of interest.

\section{References}

1. Davì, G.; Patrono, C. Platelet Activation and Atherothrombosis. N. Engl. J. Med. 2007, 357, 2482-2494. [CrossRef] [PubMed]

2. Angiolillo, D.J. ADP Receptor Antagonism. Am. J. Cardiovasc. Drugs 2007, 7, 423-432. [CrossRef] [PubMed]

3. Hollopeter, G.; Jantzen, H.M.; Vincent, D.; Li, G.; England, L.; Ramakrishnan, V.; Yang, R.B.; Nurden, P.; Nurden, A.; Julius, D.; et al. Identification of the platelet ADP receptor targeted by antithrombotic drugs. Nature 2001, 409, 202-207. [CrossRef] [PubMed]

4. Fagura, M.S.; Dainty, I.A.; McKay, G.D.; Kirk, I.P.; Humphries, R.G.; Robertson, M.J.; Dougall, I.G.; Leff, P. P2Y $\mathrm{Y}_{1}$-receptors in human platelets which are pharmacologically distinct from $\mathrm{P}^{2} \mathrm{Y}_{\mathrm{ADP}}$-receptors. Br. J. Pharmacol. 1998, 124, 157-164. [CrossRef] [PubMed]

5. André, P.; Delaney, S.M.; LaRocca, T.; Vincent, D.; DeGuzman, F.; Jurek, M.; Koller, B.; Phillips, D.R.; Conley, P.B. P2Y12 regulates platelet adhesion/activation, thrombus growth, and thrombus stability in injured arteries. J. Clin. Investig. 2003, 112, 398-406. [CrossRef] [PubMed]

6. Cattaneo, M. P2Y $\mathrm{Y}_{12}$ receptors: Structure and function. J. Thromb. Haemost. 2015, 13 (Suppl. 1), 10-16. [CrossRef] [PubMed]

7. Faria, R.; Ferreira, L.; Bezerra, R.; Frutuoso, V.; Alves, L. Action of Natural Products on P2 Receptors: A Reinvented Era for Drug Discovery. Molecules 2012, 17, 13009-13025. [CrossRef] [PubMed]

8. Hagihara, K.; Kazui, M.; Ikenaga, H.; Nanba, T.; Fusegawa, K.; Takahashi, M.; Kurihara, A.; Okazaki, O.; Farid, N.A.; Ikeda, T. Comparison of formation of thiolactones and active metabolites of prasugrel and clopidogrel in rats and dogs. Xenobiotica 2009, 39, 218-226. [CrossRef] [PubMed]

9. Franchi, F.; Angiolillo, D.J. Novel antiplatelet agents in acute coronary syndrome. Nat. Rev. Cardiol. 2015, 12, 30-47. [CrossRef] [PubMed]

10. Moon, J.Y.; Franchi, F.; Rollini, F.; Rivas Rios, J.R.; Kureti, M.; Cavallari, L.H.; Angiolillo, D.J. Role of Genetic Testing in Patients undergoing Percutaneous Coronary Intervention. Expert Rev. Clin. Pharmacol. 2017, 11, 151-154. [CrossRef] [PubMed]

11. Golwala, H.; Bhatt, D.L. The timing of P2Y12 inhibitor initiation in the treatment of ACS? Dose the evidence exist in this era? Prog. Cardiovasc. Dis. 2018, 60, 471-477. [CrossRef] [PubMed]

12. Tantry, U.; Navarese, E.P.; Myat, A.; Gurbel, P. Selection of P2Y12 inhibitor in percutaneous coronary intervention and/or acute coronary syndrome. Prog. Cardiovasc. Dis. 2018, 60, 460-470. [CrossRef] [PubMed]

13. Mega, J.L.; Simon, T.; Collet, J.P.; Anderson, J.L.; Antman, E.M.; Bliden, K.; Cannon, C.P.; Danchin, N.; Giusti, B.; Gurbel, P.; et al. Reduced-Function CYP2C19 Genotype and Risk of Adverse Clinical Outcomes Among Patients Treated With Clopidogrel Predominantly for PCI. J. Am. Med. Assoc. 2010, 304, 1821-1830. [CrossRef] [PubMed] 
14. Simon, T.; Verstuyft, C.; Mary-Krause, M.; Quteineh, L.; Drouet, E.; Méneveau, N.; Steg, P.G.; Ferrières, J.; Danchin, N.; Becquemont, L. Genetic Determinants of Response to Clopidogrel and Cardiovascular Events. N. Engl. J. Med. 2009, 360, 363-375. [CrossRef] [PubMed]

15. Wiviott, S.D.; Braunwald, E.; McCabe, C.H.; Montalescot, G.; Ruzyllo, W.; Gottlieb, S.; Neumann, F.J.; Ardissino, D.; De Servi, S.; Murphy, S.A.; et al. Prasugrel versus Clopidogrel in Patients with Acute Coronary Syndromes. N. Engl. J. Med. 2007, 357, 2001-2015. [CrossRef] [PubMed]

16. James, S.K.; Roe, M.T.; Cannon, C.P.; Cornel, J.H.; Horrow, J.; Husted, S.; Katus, H.; Morais, J.; Steg, P.G.; Storey, R.F.; et al. Ticagrelor versus clopidogrel in patients with acute coronary syndromes intended for non-invasive management: Substudy from prospective randomized PLATelet inhibition and patient Outcomes (PLATO) trial. BMJ 2011, 342, 3527. [CrossRef] [PubMed]

17. Yousuf, O.; Bhatt, D.L. The evolution of antiplatelet therapy in cardiovascular disease. Nat. Rev. Cardiol. 2011, 8, 547-559. [CrossRef] [PubMed]

18. Schoener, L.; Jellinghaus, S.; Richter, B.; Pfluecke, C.; Ende, G.; Christoph, M.; Quick, S.; Loehn, T.; Speiser, U.; Poitz, D.M.; et al. Reversal of the platelet inhibitory effect of the $\mathrm{P}^{2} \mathrm{Y}_{12}$ inhibitors clopidogrel, prasugrel, and ticagrelor in vitro: A new approach to an old issue. Clin. Res. Cardiol. 2017, 106, 868-874. [CrossRef] [PubMed]

19. Vig, B.S.; Huttunen, K.M.; Laine, K.; Rautio, J. Amino acids as promoieties in prodrug design and development. Adv. Drug Deliv. Rev. 2013, 65, 1370-1376. [CrossRef] [PubMed]

20. Sastry, T.U.; Rao, K.N.; Reddy, T.A.; Gandhi, P. Identification and Synthesis of Impurities Formed During Prasugrel Hydrochloride Preparation. Asian J. Chem. 2013, 25, 7783. [CrossRef]

21. Liu, J.A.; Guo, X.P.; Liang, S.; An, F.; Shen, H.Y.; Xu, Y.J. Regioselective synthesis of 5'-amino acid esters of some nucleosides via orthogonal protecting protocol. Tetrahedron 2015, 71, 1409-1412. [CrossRef]

22. Farid, N.A.; Smith, R.L.; Gillespie, T.A.; Rash, T.J.; Blair, P.E.; Kurihara, A.; Goldberg, M.J. The Disposition of Prasugrel, a Novel Thienopyridine, in Humans. Drug Metab. Dispos. 2007, 35, 1096-1104. [CrossRef] [PubMed]

23. Pereillo, J.M.; Maftouh, M.; Andrieu, A.; Uzabiaga, M.F.; Fedeli, O.; Savi, P.; Pascal, M.; Herbert, J.M.; Maffrand, J.P.; Picard, C. Structure and stereochemistry of the active metabolite of clopidogrel. Drug Metab. Dispos. 2002, 30, 1288-1295. [CrossRef] [PubMed]

24. Sugidachi, A.; Asai, F.; Ogawa, T.; Inoue, T.; Koike, H. The in vivo pharmacological profile of CS-747, a novel antiplatelet agent with platelet ADP receptor antagonist properties. Br. J. Pharmacol. 2000, 129, 1439-1446. [CrossRef] [PubMed]

25. Niitsu, Y.; Jakubowski, J.A.; Sugidachi, A.; Asai, F. Pharmacology of CS-747 (Prasugrel, LY640315), a Novel, Potent Antiplatelet Agent with in Vivo P2Y12 Receptor Antagonist Activity. Semin. Thromb. Hemost. 2005, 31, 184-194. [CrossRef] [PubMed]

26. Jakubowski, J.A.; Winters, K.J.; Naganuma, H.; Wallentin, L. Prasugrel: A Novel Thienopyridine Antiplatelet Agent. A Review of Preclinical and Clinical Studies and the Mechanistic Basis for Its Distinct Antiplatelet Profile. Cardiovasc. Drug Rev. 2007, 25, 357-374. [CrossRef] [PubMed]

27. Velder, J.; Hirschhäuser, C.; Waldmann, C.; Taubert, D.; Bouman, H.J.; Schmalz, H.G. A Scalable Synthesis of ( \pm )-2-Oxoclopidogrel. Synlett 2010, 3, 467-469. [CrossRef]

28. Born, G.V.R. Aggregation of blood platelets by adenosine diphosphate and its reversal. Nature 1962, 194, 927-929. [CrossRef] [PubMed]

29. Dejana, E.; Callioni, A.; Quintana, A.; de Gaetano, G. Bleeding time in laboratory animals II—A comparison of different assay conditions in rats. Thromb. Res. 1979, 15, 191-197. [CrossRef]

Sample Availability: Samples of the compounds reported in this paper are available from the authors. 